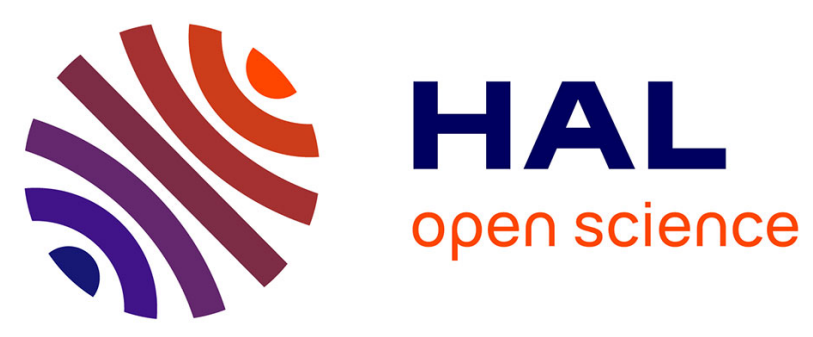

\title{
Insight into the formation and stability of solid electrolyte interphase (SEI) for nanostructured siliconbased anode electrodes used in Li-ion batteries
}

Mariam Ezzedine, Mihai-Robert Zamfir, Fatme Jardali, Lucie Leveau, Eleonor

Caristan, Ovidiu Ersen, Costel Sorin Cojocaru, Ileana Florea

\section{To cite this version:}

Mariam Ezzedine, Mihai-Robert Zamfir, Fatme Jardali, Lucie Leveau, Eleonor Caristan, et al.. Insight into the formation and stability of solid electrolyte interphase (SEI) for nanostructured siliconbased anode electrodes used in Li-ion batteries. ACS Applied Materials \& Interfaces, Washington, D.C. : American Chemical Society, 2021, 13 (21), pp.24734-24746. 10.1021/acsami.1c03302 . hal-03322747

\section{HAL Id: hal-03322747 \\ https://hal.archives-ouvertes.fr/hal-03322747}

Submitted on 19 Aug 2021

HAL is a multi-disciplinary open access archive for the deposit and dissemination of scientific research documents, whether they are published or not. The documents may come from teaching and research institutions in France or abroad, or from public or private research centers.
L'archive ouverte pluridisciplinaire HAL, est destinée au dépôt et à la diffusion de documents scientifiques de niveau recherche, publiés ou non, émanant des établissements d'enseignement et de recherche français ou étrangers, des laboratoires publics ou privés. 


\title{
Insight into the formation and stability of solid electrolyte interphase (SEI) for nanostructured silicon- based anode electrodes used in Li-ion batteries
}

\author{
Mariam EZZEDINE ${ }^{1}$, Mihai-Robert ZAMFIR ${ }^{1,2}$, Fatme JARDALI $^{1}$, Lucie LEVEAU $^{1,3}$ \\ Eleonor CARISTAN ${ }^{1}$, Ovidiu ERSEN ${ }^{4}$, Costel-Sorin COJOCARU ${ }^{1}$, Ileana FLOREA ${ }^{1, \text { * }}$
}

${ }^{1}$ LPICM, CNRS, Ecole polytechnique, IP Paris, 91228 Palaiseau Cedex, France

${ }^{2}$ National Institute for Laser, Plasma \& Radiation Physics (INFLPR), Atomistilor Street, No. 409, Magurele, Ilfov RO-077125, Romania

${ }^{3}$ Renault SAS, DREAM/DETA/SEE, 1, Avenue du Golf, 78288 Guyancourt, France

${ }^{4}$ Institut de Physique et Chimie des Matériaux de Strasbourg (IPCMS), UMR 7504 CNRS Université de Strasbourg, 23 rue du Loess, 67034 Strasbourg Cedex 2, France

*lenuta-ileana.florea@polytechnique.edu

\begin{abstract}
Silicon-based anode fabrication with nano-scale structuration improves the energy density and life cycle of Li-ion batteries. As-synthesized silicon ( $\mathrm{Si}$ ) nanowires (NWs) or nanoparticles (NPs) directly on the current collector represent a credible alternative to conventional graphite anode. However, the operating potentials of these electrodes are below the electrochemical stability window of all electrolytes used in commercial Li-ion systems. During the first charging phase of the cell, partial decomposition of the electrolyte takes place, which leads to the formation of a layer at the surface of the electrode, called Solid Electrolyte Interphase (SEI). A stable and continuous SEI layer formation is a critical factor to achieve reliable life-time stability of the battery. Once formed, the SEI acts as a passivation layer that minimizes further degradation of the electrolyte during cycling, while allowing lithium ions diffusion with their subsequent insertion into the active material and ensures reversible operation of the electrode. However, one of the major issues requiring deeper investigation is the assessment of the morphological extension of the SEI layer into the active material which is one of the main parameters affecting the anode performances. In the present study, we use electron tomography (ET) with low electron dose to retrieve three-dimensional information on the SEI layer formation and its stability around SiNWs and SiNPs. The possible mechanisms of the SEI evolution could be inferred from the interpretation and analysis of the reconstructed volumes. Significant volume variations in the SiNW and an inhomogeneous distribution of the SEI layer around the NWs are observed during cycling and provide insights of the potential mechanism leading to the generally reported SiNWs anodes capacity fading. By contrast, analysis of the reconstructed SiNPs' volume for a sample undergoing one lithiation-delithiation cycle evidence that the SEI remains homogeneously distributed around the NPs that retain their spherical morphology and points to the potential benefit of such nanoscale Si anode materials to improve their cycling lifetime.
\end{abstract}




\section{Introduction}

The demand for rechargeable power sources with high electrochemical performances is growing steadily and correlates with the society's ever-increasing need for developing a next generation of lithium-ion batteries (LiBs). Electric vehicles and grid energy storage increasingly monopolize the LiBs market demands. Accordingly, considerable efforts are devoted by industry and academia actors to develop high capacity and long-cycle life electrode materials at lower cost. ${ }^{1-3}$ Carbonaceous anode materials, such as for instance traditional graphite, a component used in conventional commercial LiBs, offers a limited theoretical capacity of $372 \mathrm{mAh} \mathrm{g}^{-1}$. $^{4,5}$ The main goal for next-generation LiBs is to promote new electrode materials with better electrochemical properties, including (i) a high theoretical specific capacity, (ii) a fast $\mathrm{Li}^{+}$-ion diffusion path for improving the intercalation/ de-intercalation rate, and (iii) a stable structure. ${ }^{6}$

Among the various anode materials studied in literature, silicon ( $\mathrm{Si}$ ) has attracted extensive attention due to its high theoretical capacity of $4200 \mathrm{mAh} \mathrm{g}^{-1}$ and its abundance in the earth's crust. ${ }^{7}$ The main drawback for use of $\mathrm{Si}$ as a LiB electrode relates to the dramatic volume variation (up to $300 \%$ ) upon cycling, leading to the creation of microcracks and to severe pulverization of the material. This results in a degradation of the battery performance, hindering its potential commercialization. ${ }^{8,9}$

To hamper these shortcomings, new designs of Si structures have been proposed, such as thin films ${ }^{10,11}$, hybrid structures ${ }^{12-17}$, composites ${ }^{18,19}$ and nanostructured Si-based materials. ${ }^{20-27}$ In general, replacing a bulk material with a nanostructured one helps mitigation of the volume changes of the active material and, thus, tempers the mechanical pulverization and subsequent structural degradation. Accordingly, designing nanostructured Si anodes, such as nanowires (NWs) ${ }^{28-32}$ or nanoparticles (NPs), ${ }^{32-37}$ can dramatically improve the electrochemical performances of the battery and may become a credible alternative to graphite. 
Indeed, NWs have attracted great attention for their application as anodes in LiBs due to several advantages provided by their specific morphology. The one-dimensional (1-D) structure allows the accommodation of the important volume changes during repeated lithiation/ delithiation processes without cracking. Moreover, the structure offers a direct 1-D electronic pathway for rapid charge transport and affords effective lithium insertion and extraction due to the increased specific surface area for $\mathrm{Li}^{+}$-ions exchange. Additionally, direct synthesis of NWs on a current collector highly improves the electrical conduction of the electrode. ${ }^{28,38}$

On the other side, the recent development of new nanoparticles anode design is gathering increased attention because the volume variation and the pulverization issues of the Si anode could be effectively buffered by downsizing the Si particle to a nanometric scale. ${ }^{24,39}$ An alternative electrode design that includes both silicon nanoparticles (SiNPs) and vertically aligned carbon nanotubes (VACNTs) has recently gained a lot of interest thanks to its ability for improving the overall charge capacity in LiBs. 1-D directional-electron-transport through VACNTs anchored on top of a macroscopic substrate ensures an optimal current collection for large assemblies of SiNPs thus resulting in high active material areal loading. On one hand silicon at nanoscale enhances the accommodation of the large volume changes without mechanical degradation and on the other hand the VACNTs conductive matrix eliminates the use of carbon additives or binders which add additional weight and lower the overall specific capacity of the electrode. ${ }^{14,40,41}$ Also, it is important to note that the fabrication of VACNTs decorated with SiNPs (i.e. SiNPs@VACNTs) electrode is compatible with the roll-to-roll manufacturing techniques and can allow large-scale production of Si anodes for LiBs. ${ }^{42}$

Another major drawback for the use of any Si-based electrode in commercial LiBs is related to the instability of the Solid Electrolyte Interface (SEI) layer formation ${ }^{43}$ and improving the electrode design thus remains a challenge. In this context, probing the reaction kinetics and the microstructural evolution during battery operation may provide extremely useful information 
for the formation of the SEI layer. A thorough investigation of the morphological evolution of the SEI on SiNWs and SiNPs is mandatory and will allow a clear understanding of the role of the material architecture, with the final goal of the improvement of the electrode design.

Amongst the various characterization techniques, such as spectroscopic ellipsometry, ${ }^{44}$ X-ray photoelectron spectroscopy (XPS), nuclear magnetic resonance (NMR), ${ }^{45-47} \mathrm{X}$-ray diffraction (XRD) ${ }^{48}$ or in situ electrochemical impedance spectroscopy, ${ }^{49}$ transmission electron microscopy (TEM)-based techniques retain clear advantages as most suited characterizations tools due to the possibility to directly visualize the morphology and the internal structure of the LiBs electrodes. TEM may not only provide information on the structure of the material under study, but also on its chemical and morphological characteristics. During the last decades, many TEM-based studies focused on understanding the mechanisms of lithium insertion in Si-based materials by using generally conventional, two-dimensional (2D) analysis. However, scarce studies focused on the assessment of the exact morphology of the SEI layer. The reason for such limited number of research studies may be explained by the inherent fragility of the layer once exposed to the electron beam inside the microscope. The SEI layer morphology has been however investigated using the SEM technique. ${ }^{50,51}$ Other groups used more advanced in situ TEM techniques to mimic the behavior of different anodes materials at a nanometeric scale. The pioneering work of Huang et al. has demonstrated for the first time the "open-cell" concept creating a nano - battery inside the TEM for the observation of the microstructural evolution of $\mathrm{SnO}_{2}$ nanowires during the electrochemical lithiation. The concept was later extended for the study of other anode materials behavior. ${ }^{8,52-57}$

In order to understand, from a morphological point of view, the process of SEI formation and its morphology evolution in the case of more complex nanostructures such as for instance SiNWs or SiNPs@VACNTs heterostructures, the analysis of classical 2D projection images is insufficient. To this regard, over the past few years, electron tomography has become 
a standard technique to visualize in three dimensions (3D) the morphology, the internal structure or the chemical composition of a wide variety of nanostructures ${ }^{58-61}$ and for an extended range of electrode materials. ${ }^{62}$ The approach is based on the acquisition of a series of 2D projection images collected at different tilt angles over a large angular range. ${ }^{63}$ Moreover, the continuous technological advancement made possible the design of specific sample holders, such as vacuum TEM holders, cryo TEM holders or in situ holders, ${ }^{64,65}$ that reach the tilt range required for electron tomography, thus allowing for 3D studies of sensitive materials like the electrochemically cycled SiNWs or SiNPs, without air exposure or severe beam damaging.

In this general framework of development of new materials and associated characterization tools, the aim of the present work is to provide valuable observational evidence on the formation and stability of solid electrolyte interphase (SEI) for two classes of nanostructured Si-based materials which are explored as anode electrodes in LiBs. With this objective, electron tomography studies were carried out on several NWs having undergone three lithiation/ delithiation cycles at similar delithiation cut-off voltages and SiNPs having undergone one complete lithiation/ delithiation cycle.

\section{Methods}

\subsection{Synthesis of the SiNWs and the hybrid SiNPs@VACNTs electrodes}

\subsubsection{Synthesis of SiNWs electrodes}

SiNWs were synthesized by a Hot-Filament assisted Chemical Vapor deposition (HF-CVD) method according to the vapor-liquid-solid (VLS) growth mechanism. ${ }^{47}$ The growth was conducted directly on a stainless-steel current collector using gold ( $\mathrm{Au})$ as catalyst. Prior to HFCVD growth of SiNWs, a $3 \mathrm{~nm}$ thin Au film was deposited on the substrate by electron beam evaporation. During the VLS growth, the metal catalytic film was heated to $540{ }^{\circ} \mathrm{C}$ and the 
furnace was filled with $\mathrm{H}_{2}$ at a flow rate of $100 \mathrm{sccm}$ for 2 minutes of pre-treatment to transform the Au thin film into clusters. A tungsten filament was powered at $130 \mathrm{~W}$ to dissociate the hydrogen $\left(\mathrm{H}_{2}\right)$ molecules. Then, silane $\left(\mathrm{SiH}_{4}\right)$ gas was introduced into the furnace at a flow rate of $20 \mathrm{sccm} . \mathrm{SiH}_{4}$ was mixed with the carrier gas $\mathrm{H}_{2}$ and the pressure was maintained at 7.5 mbar during the 30 minutes growth of the $\mathrm{SiNWs}$. When the $\mathrm{SiH}_{4}$ molecules adsorb on the surface of the catalyst droplets, they dissociate to form atomic Si which diffuses within the drop, forming a liquid Au-Si alloy. As more $\mathrm{Si}$ atoms are added, the catalyst droplets achieve supersaturation at the eutectic mixture and precipitation occurs at the solid-liquid interface. A dynamic equilibrium is rapidly achieved between the continuous $\mathrm{Si}$ atoms incorporation in the liquid eutectic drop and the Si atoms precipitating at the liquid-solid interface that subsequently nucleate the solid crystalline Si phase in form of a 1-D nanostructure, i.e. SiNW.

\subsubsection{Synthesis of SiNPs electrodes}

A two-stage process was adopted for the synthesis of the hybrid SiNPs@VACNTs electrodes. Prior to the CNTs growth, a $50 \mathrm{~nm}$ aluminum oxide $\left(\mathrm{Al}_{2} \mathrm{O}_{3}\right)$ barrier layer followed by a $5 \mathrm{~nm}$ thick layer of iron $(\mathrm{Fe})$ were deposited on a commercial Copper $(\mathrm{Cu})$ foil by molecular beam evaporation (MBE) under high vacuum $\left(10^{-9}\right.$ mbar). The CNTs growth was carried out by a double-hot-filament-assisted chemical vapor deposition dHF-CVD technique. ${ }^{66}$ This technique allows the growth of straight, vertically aligned CNTs forests over a large area with excellent uniformity in diameter, length and density. Before injecting the precursor gas molecules (i.e. hydrogen $\left(\mathrm{H}_{2}\right)$ and methane $\left(\mathrm{CH}_{4}\right)$ ) for the growth of CNTs, the system was heated to the desired temperature $\left(600^{\circ} \mathrm{C}\right)$. A pretreatment step of the sample under pure, activated $\mathrm{H}_{2}$ was performed at a flow rate of $100 \mathrm{sccm}$, pressure of $50 \mathrm{mbar}$ and filament power of $180 \mathrm{~W}$ for 5 minutes in order to dewet the catalyst layer into small Fe NPs. Finally, a gas mixture of $\mathrm{H}_{2} / \mathrm{CH}_{4}$ was added into the reactor for 30 minutes at a pressure of 50 mbar. The flow rate of $\mathrm{H}_{2}$ was 50 sccm and the filament power was $180 \mathrm{~W}$, while the flow rate of $\mathrm{CH}_{4}$ was $50 \mathrm{sccm}$ and the 
filament power was $150 \mathrm{~W}$. The addition of $\mathrm{H}_{2}$ during the CNTs growth is essential as it acts as an etching agent, limiting parasitic amorphous carbon (a-C) deposition.

Subsequently, in a second step, the deposition of SiNPs on the as-grown CNTs forest was carried out using the previously described (SiNWs synthesis) HF-CVD process using a mixed gas flow of $\mathrm{H}_{2}$ and $\mathrm{SiH}_{4}$ at $540{ }^{\circ} \mathrm{C}$. The sample was loaded into the reactor preheated at $540{ }^{\circ} \mathrm{C}$ and filled with $\mathrm{H}_{2}$ gas. After 5 minutes, the $\mathrm{SiH}_{4}$ gas was introduced into the reactor at a flow rate of $10 \mathrm{sccm}$ while maintaining a $\mathrm{H}_{2}$ gas flow rate of $30 \mathrm{sccm}$. The tungsten filament placed at the $\mathrm{H}_{2}$ gas inlet was heated to $72 \mathrm{~W}$ (approx. $1000{ }^{\circ} \mathrm{C}$ ) in order to etch the excess $\mathrm{Si}$ on the surface of the CNTs. This can help the SiNPs to embed easily onto the CNTs walls. The pressure was maintained at 7 mbar for the 6 minutes synthesis duration.

\subsection{Electrochemical Characterization}

The samples considered in the present work were electrochemically cycled in half-cells configuration using metallic lithium foil as counter electrode and either SiNWs or SiNPs@VACNTs as working electrode. The coin cells were mounted in an argon-filled glove box, using home-made electrolyte prepared by mixing $1 \mathrm{M}$ lithium hexafluorophosphate (LiPF6) (1:1 by volume) with ethylene carbonate (EC) and dimethyl carbonate (DMC) as well as fluoroethylene carbonate (FEC) as additive (5wt\%). The FEC additive was chosen because it enhances the cycling stability of Si-based electrodes, ${ }^{47}$ and because the SEI layer formed with this electrolyte exhibited good stability under a low intensity electron beam (see Figure S1 in Supporting Information).

For electron tomography characterizations, the lithiation of the SiNWs electrode was carried out down to $20 \mathrm{mV}$ and the delithiation cut-off voltages were chosen to be $0.6,0.8$ and $2 \mathrm{~V}$ vs $\mathrm{Li}^{+} / \mathrm{Li}$ at $\mathrm{C} / 20$ current rate. For the SiNPs@VACNTs hybrid electrode, the cell was lithiated up to $20 \mathrm{mV}$ and the delithiation cut-off voltage was $2 \mathrm{~V} \mathrm{vs} \mathrm{Li}^{+} / \mathrm{Li}$ at $\mathrm{C} / 20$. The cycling of the coin cells was interrupted at the delithiated state and transferred to the glove box for disassembling 
under argon environment in order to avoid any air contamination of the sample. The electrodes were rinsed with DMC and dried in the glove box.

\subsection{D-TEM and electron tomography characterization}

For the 2D-TEM observations and the electron tomography studies, in order to avoid any air exposure, the cycled SiNWs and SiNPs@VACNTs were recovered from the coin cell electrode inside the glove box. Once the coin cell disassembled and the anode electrode rinsed with DMC the SiNWs and the SiNPs@VACNTs were deposited on the TEM Cu grid supporting a very thin carbon membrane and on high tilt sample holder. Thereafter, the transfer to the TEM microscope of this later was performed using plastic containers previously sealed inside the glove box. Before insertion within the TEM chamber the containers were unsealed under argon flow. The TEM observations were performed at low electron dose to avoid eventual irradiation damages which could lead to the degradation of the SEI layer. The electron dose used for the $2 \mathrm{D}$ images was about $120 \mathrm{e} / \mathrm{A}^{2} \mathrm{~s}^{-1}$. For the tomography studies, the acquisition of the tilt series was performed in the classical Bright-Field (BF) mode of the JEOL 2100F microscope, using the GATAN acquisition software and a 2048x2048 pixels cooled CCD array detector. The tomography plug-in allows for an automatic variation of the tilt angle step by step, a correction of the focus of the image and the preservation of the object under study within the field of view. The tilt angle was varied in a range of $-70^{\circ}$ to $+70^{\circ}$, with an image recorded every $2^{\circ}$ using the Saxton scheme giving a total of 100 images with a total acquisition time of about 40 minutes using a total dose of $1200 \mathrm{e} / \mathrm{A}^{2} \mathrm{~s}^{-1}$. The data treatment of the tilt series for preliminary image processing procedure was performed using the IMOD software. ${ }^{67}$ The volumes reconstruction were obtained using 15 iterations of the algebraic reconstruction technique algorithm (ART) ${ }^{68}$ implemented in the TOMOJ software. ${ }^{69}$ Using the experimental acquisition parameters and the specimen characteristics and using the relations by Midgley et al. ${ }^{70}$ the spatial resolution of the reconstruction was estimated to be around $1.5 \mathrm{~nm}$ in the directions perpendicular to the electron 
beam. In the parallel direction, the resolution is worsened by a factor of 1.1 due to the limited maximum tilt angle. Generally, we consider the information limit of 3D analysis to be about 2 $\mathrm{nm}$ in our case. To model the computed volume, we finally used a segmentation procedure based on the grey-level intensities of the voxels, followed by surface rendering methods. More details on the experimental set-up, volume reconstruction and analysis can be found elsewhere. ${ }^{71,72}$

\section{Results and Discussions}

\subsection{SiNWs anode material}

\subsubsection{General characteristics of SiNWs anode material}

Figure 1 shows SEM and TEM images of the SiNWs before and after cycling. Figure 1A shows a high magnification SEM micrograph of the pristine SiNWs film grown using the CVD approach. As it can be observed, the film is very dense and consists of long and entangled NWs presenting similar general morphology. The average length, determined by statistical analysis of more than $200 \mathrm{NWs}$, is $2 \mu \mathrm{m}$ while their observed diameter range is between 50 and $60 \mathrm{~nm}$. From the TEM analyses, we can deduce that most of the NWs present a high crystalline order as evidenced by the corresponding diffraction pattern shown in the inset of Figure 1A. We could also distinguish the presence of the remaining gold catalyst droplets at the tip of the SiNWs (see Figure 1A). Concerning the SiNWs characteristics after cycling, significant morphological changes of the NWs can be observed. As expected, the NWs undergo expansion during $\mathrm{Li}^{+}$-ion insertion and their diameter expanded up to $0.2-0.3 \mu \mathrm{m}$. Another typical finding in relation with the volume expansion is the evidence of the SEI layer formation due to the partial decomposition of the electrolyte. Typically, the SEI layer covered completely the NW surface which evolved from a crystalline structure to an amorphous one (See Figure S1 in Supporting Information). 


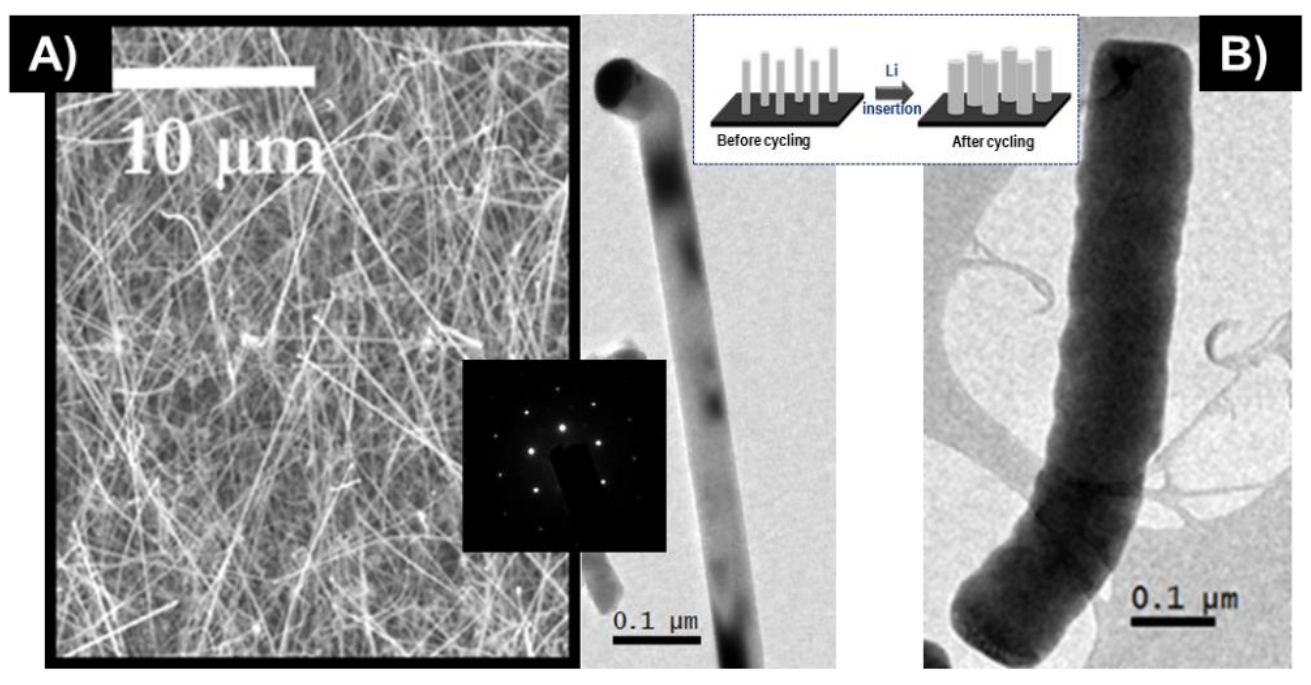

Figure 1. SEM and TEM analyses of SiNWs before (A) and after (B) the electrochemical cycles. Inset of figure (A) an electron diffraction pattern of a SiNW illustrating its highly crystalline structure. Second inset of the figure contains a schematically representation illustrating the SiNWs volume expansion after the $\mathrm{Li}$ insertion is also given

Previous studies have suggested that the modification of the electrochemical cycling parameters can improve the life cycle of the SiNWs electrode. Thus, a considerable enhancement of the cycling stability can be obtained either by limiting the lithiation/ delithiation capacities to restrain the Si material volume variation or by limiting the lithiation capacity with a reduced delithiation cut-off voltage. ${ }^{47}$ When applying this cycling strategy, the shape of the galvanostatic cycles performed at $1 \mathrm{C}$ rate with a limited lithiation capacity of 900 $\mathrm{mAh} \mathrm{g}^{-1}$ combined with different upper cut-off voltages of delithiation are displayed in Figure 2A. It has been found that this limited capacity can be maintained for 610,1450 and 2300 cycles for delithiation cut-off voltages of $0.6,0.8$ and $2 \mathrm{~V}$, respectively. ${ }^{47}$ The origin of this enhanced stability has not been clearly identified; however one reasonable hypothesis is that it could be related to the SEI morphology and structure. Thus, a complete 2D and 3D TEM study has been performed for accessing chemical and morphological characteristics of the electrode material and to understand the improvement obtained with a reduced delithiation cut-off voltage. Figure 2 shows the TEM images recorded in the low dose mode on the SiNWs electrodes cycled at delithiation cut-off voltages of $0.6,0.8$ and $2 \mathrm{~V}$ while keeping the number of cycles constant at 
3. Different contrast regions can be easily distinguished at the surface of the studied NWs. The images show that the NWs having undergone delithiation cut-off voltages of $0.8 \mathrm{~V}$ and $2 \mathrm{~V}$ are characterized by similar morphologies at the central region of the NW. By contrast, an inhomogeneous SEI layer with a thickness of $83 \mathrm{~nm}$ and $72 \mathrm{~nm}$ for $0.8 \mathrm{~V}$ and $2 \mathrm{~V}$, respectively, covering the surface of the SiNWs, can be observed. As for the NWs having undergone a delithiation cut-off voltage of $0.6 \mathrm{~V}$, an additional lower contrast region can be identified from the 2D-TEM images (as indicated by white arrows in Figure 2). Such light contrast or low density region can be related to the formation of a void between the SiNW and the as-formed SEI layer during the delithiation step where the extraction of the $\mathrm{Li}^{+}$-ions takes place, suggesting thus an irregular/ porous SEI layer formation. Another notable observation revealed from this 2D-TEM analysis concerns the amorphous structure of the analyzed cycled NWs that additionally confirms that they were actively subjected on the lithiation / delithiation process (see also Figure S1 in Supporting Information). A striking observation originating also from the 2D-TEM micrographs concerns the apparent thickness of the SEI layer as compared to the estimated diameter of the NWs. It is thus observed that NWs diameter increases whenever the electrode is submitted to a higher cycling number, resulting in an irregular accumulation of the SEI around their surface and is accompanied with the establishment of more irregular wire morphology (See Figure S2 in Supporting Information). 

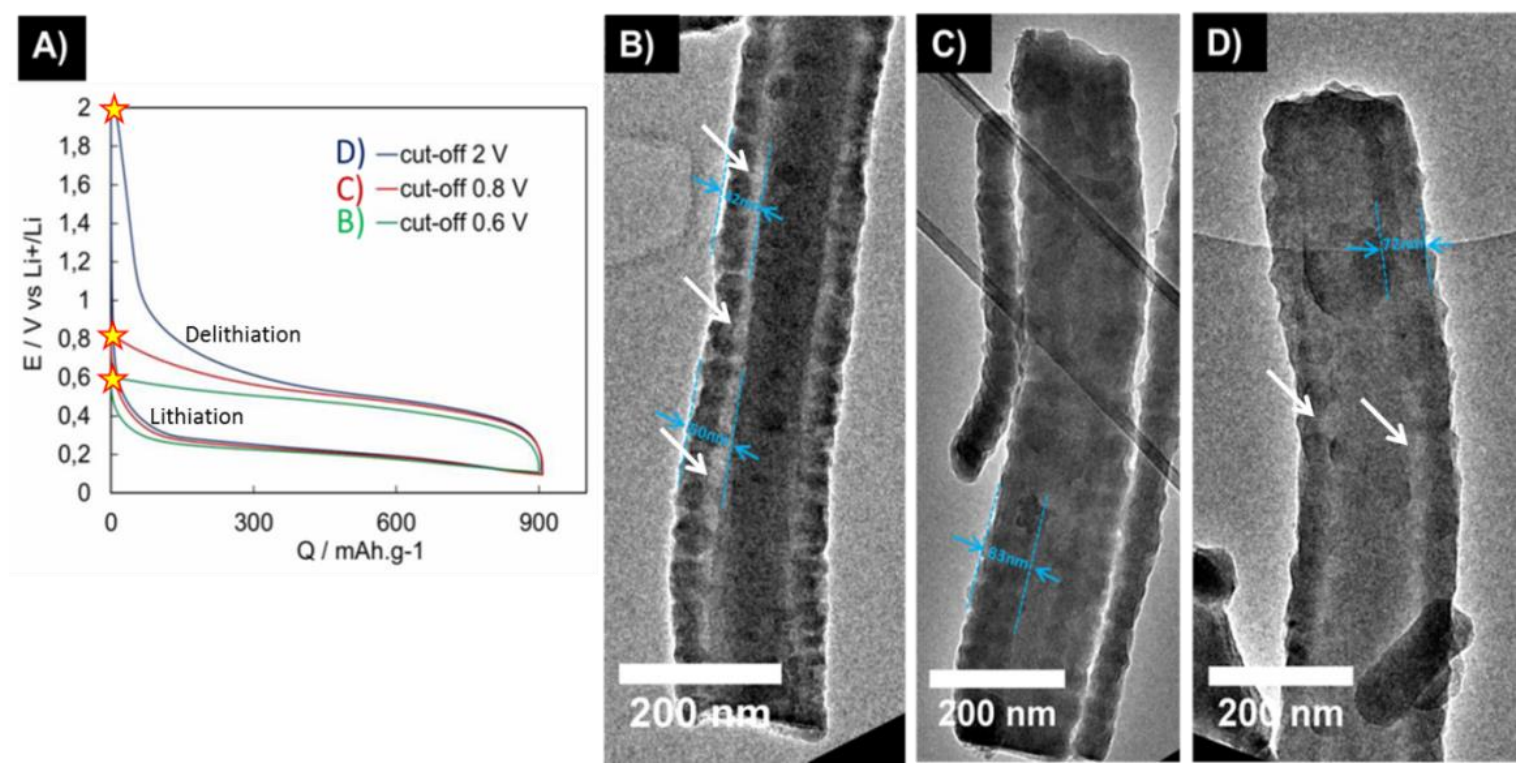

Figure 2. A) Cycling at $1 \mathrm{C}$ of SiNWs electrode vs $\mathrm{Li}$ in $\mathrm{EC} / \mathrm{DMC}+\mathrm{LiPF}_{6} 1 \mathrm{M}+\mathrm{FEC} 5 \mathrm{wt} \%$. Lithiation was done to $900 \mathrm{mAh} \mathrm{g}^{-1}$ and delithiation to $0.6 \mathrm{~V}, 0.8 \mathrm{~V}$ and $2 \mathrm{~V}$. Shape of the third charge/discharge cycle. 2D-TEM micrographs of different SiNWs cycled using the delithiation cut-off voltages represented in (A) and using the same cycling numbers B) $0.6 \mathrm{~V}-3$ cycles, C) $0.8 \mathrm{~V}-3$ cycles and D) $2 \mathrm{~V}-3$ cycles.

\subsubsection{Morphology of SiNWs anode materials by 3D-TEM}

In order to reveal the spatial distribution of the SEI layer with respect to the initial SiNWs structure and to confirm the assumptions made from the 2D-TEM observations as illustrated in Figure 1, electron tomography (ET) analyses were performed on several NWs having undergone three lithiation/ delithiation cycles at similar delithiation cut-off voltages as those illustrated in Figure 2. More exactly, for each delithiation cut-off three representative SiNWs objects were considered for ET studies. First, an ET study was performed on NWs cycled with a delithiation cut-off voltage of $0.6 \mathrm{~V}$. Figure 3A displays two classical 2D-TEM micrographs of the as-cycled NWs, extracted from the tilt series used to reconstruct the volume, on which one can easily identify two different contrast regions corresponding to the SiNW and respectively the surrounding SEI layer with a marked apparent roughness. By analyzing the projection series recorded for three different NWs (see Figure 3A and multimedia file MovieS1 and MovieS2 in Supporting Information), one can easily observe that the morphology of these 
cycled NWs exhibits a marked difference at high tilt angles in respect to that at low tilt angles (see Figure 3A). We can estimate a NW diameter of $90 \mathrm{~nm}$ at $-70^{\circ}$ tilt whilst the estimated diameter is $142 \mathrm{~nm}$ at $0^{\circ}$ and $+45^{\circ}$ tilt, with a certain roughness at NW's surface which can reasonable be originated from the presence of the as formed SEI layer. A more detailed analysis of the projections recorded at low tilt angles, on which the presence of the two contrasts is clearly visible, allow us to unambiguously conclude, that the NWs are covered by a SEI layer on both sides with a thickness that can be estimated to about $35 \mathrm{~nm}$. Representative sections through the volume of the NW obtained by reconstruction are presented in Figure 3B. A quick visualization of the volume slice by slice clearly points out to an inhomogeneous distribution of the SEI layer around the NW. This important observation is even more obvious when the volume is visualized in the direction perpendicular to the NW axis. We can clearly observe that the SEI layer is not entirely covering the NW, as suggested by the $2 \mathrm{D}$ projections. Also, very importantly, that this layer is not in direct contact with the NW along its entire periphery but remains attached by two preferential anchorage points symmetrically situated with respect to the tube axis, indicated by the yellow arrows in the $\mathrm{XZ}$ slices. Furthermore, a quantitative analysis of the reconstructions allowed us to estimate the void gap between the NW and the asformed SEI layer to about $15 \mathrm{~nm}$. Figure 3C shows a 3D model of the analyzed NW, where the red part corresponds to the SiNW and the blue part corresponds to the SEI layer. This model highlights the irregular distribution of the SEI layer around the NW as well as its external and internal roughness. Additionally, from a quantitative point of view by counting the pixels contained within the as-formed SEI layer part we were able to estimate a total volume of about $34.22 * 10^{6} \mathrm{~nm}^{3}$ covering the 2 sides of the SiNW. By analyzing the reconstructed volume, i.e., by selecting the pixels contained at the interface of the SEI surface with the SiNW we were able to determine the total contact surface between the two components to be about $0.11910^{6}$ $\mathrm{nm}^{2}$ 

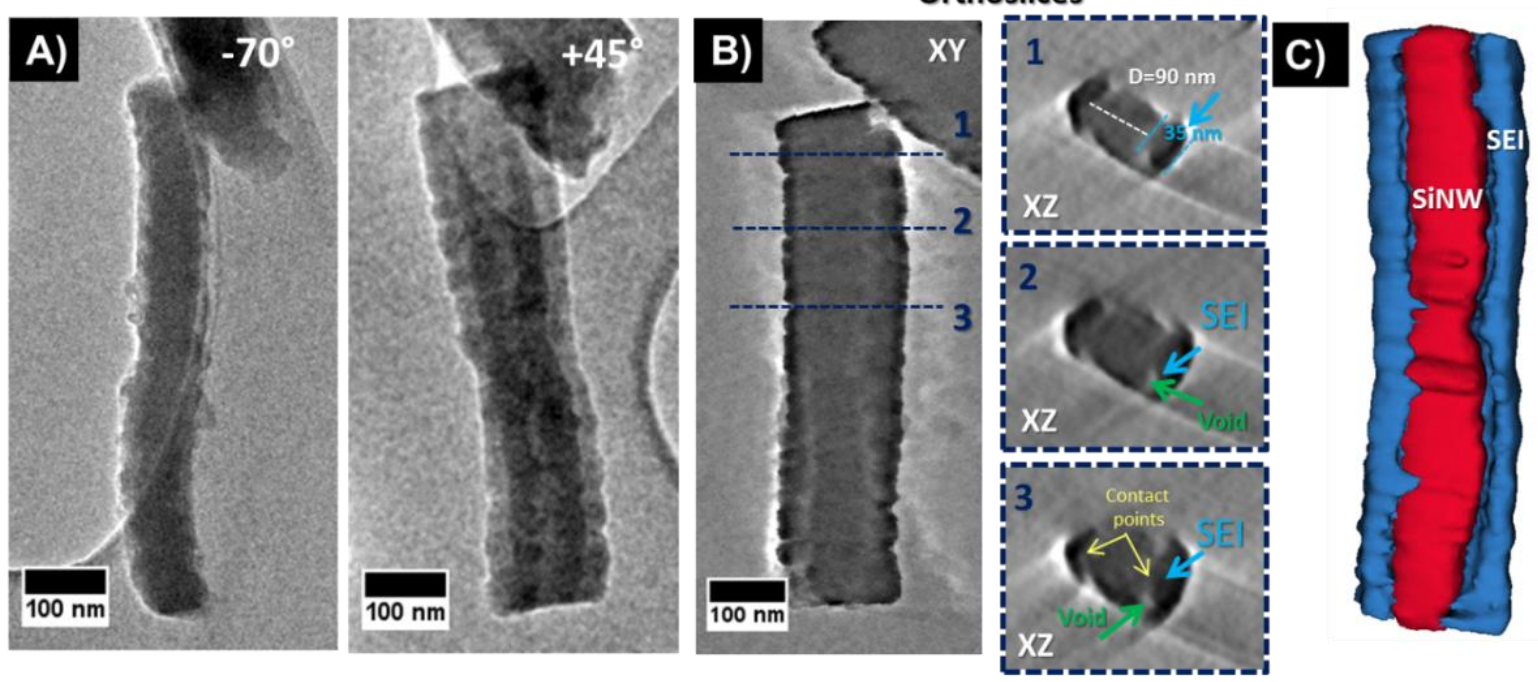

Figure 3. Electron tomography analyses of SiNW cycled using a delithiation cut-off voltage of $0.6 \mathrm{~V}$ that undergone 3 cycles. A) Projections acquired at two different tilt angles $\left(-70^{\circ}\right.$ and $\left.+45^{\circ}\right)$ from the tilt series used to calculate the reconstruction. B) Transversal and longitudinal slices acquired at different depths and orientations through the reconstructed volume, illustrating that the SEI is not fully covering the SiNW. C) 3D Model of the analyzed NW; the NW is represented in red and the SEI layer in blue.

Similar ET studies were performed on several NWs resulting from electrodes that have undergone delithiation cut-off voltage of $0.8 \mathrm{~V}$ and $2 \mathrm{~V}$ respectively. The results for two representative NWs are illustrated in Figure $4 \mathrm{~A}-\mathrm{C}$ for $0.8 \mathrm{~V}$ and Figure $4 \mathrm{D}-\mathrm{F}$ for $2 \mathrm{~V}$ delithiation cut-off voltages (see also Supporting Information multimedia file Movie S3, S4, S5, S6). SiNWs with diameters of about $110 \mathrm{~nm}$ and $220 \mathrm{~nm}$ for 0.8 and $2 \mathrm{~V}$, respectively, can be observed which also appear to be covered with a SEI layer of irregular thickness. The sliceby-slice analysis of the corresponding reconstructed volumes in the direction perpendicular to the electron beam allowed us to estimate a maximum SEI layer thickness of about $45 \mathrm{~nm}$ for the NW that had undergone delithiation cut-off voltage of $0.8 \mathrm{~V}$ and $40 \mathrm{~nm}$ for the one that undergone delithiation cut-off voltage of at 2 V, respectively (see Figure 4B and E). 

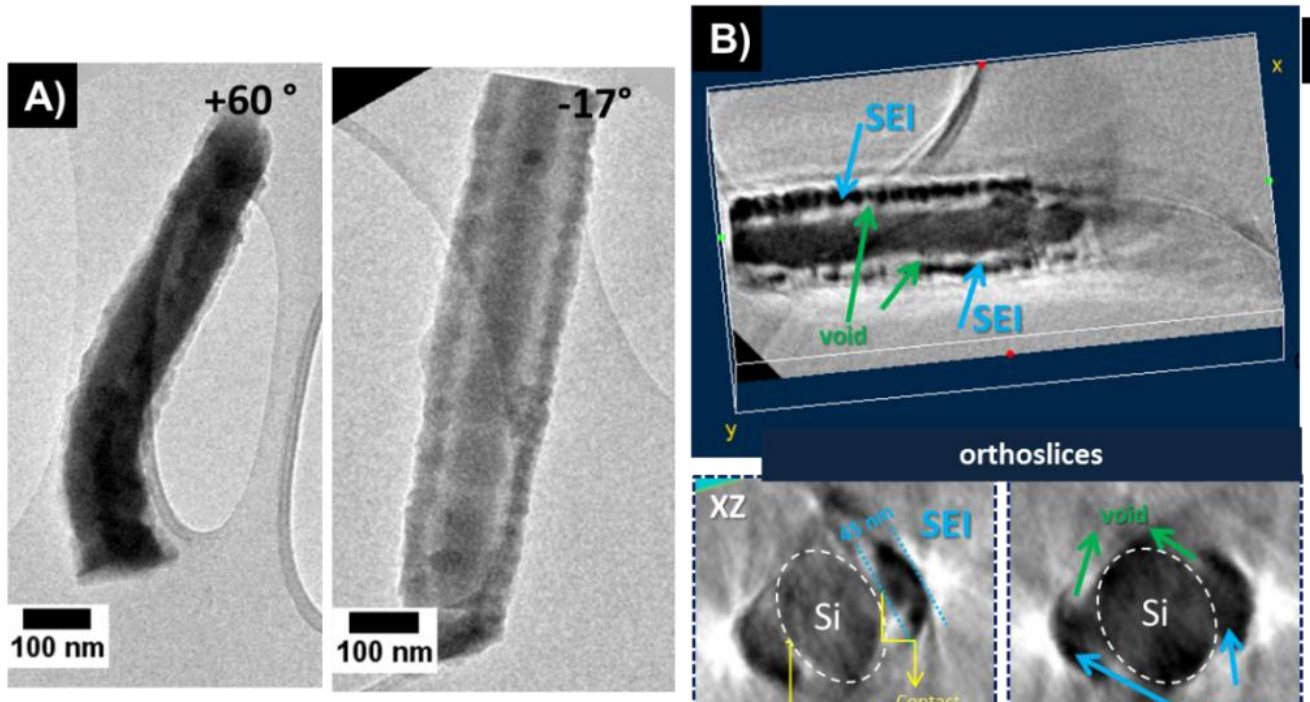

\section{C)}
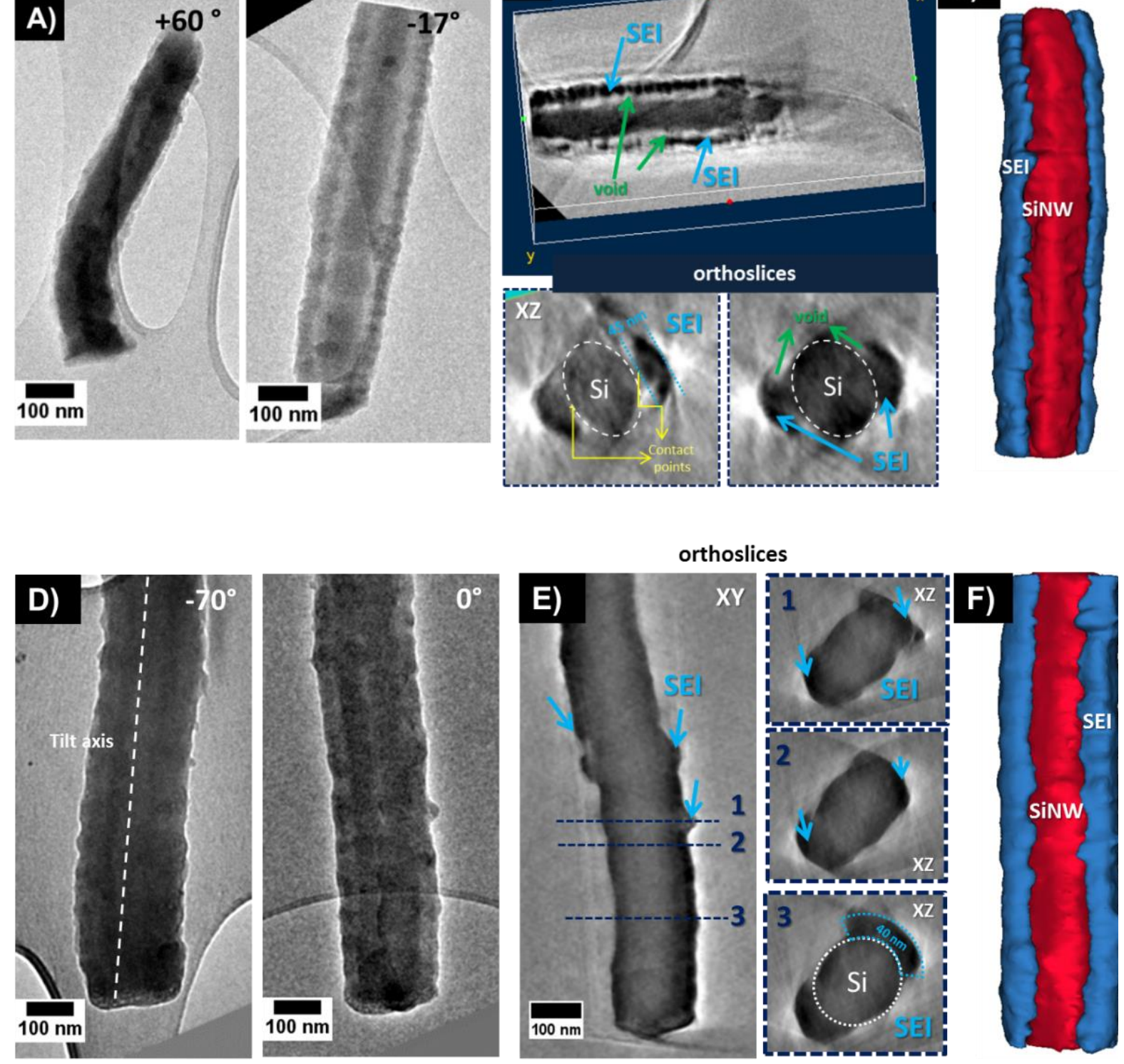

Figure 4. Electron tomography analyses of the SiNWs cycled using a delithiation cut-off voltages of $0.8 \mathrm{~V}(\mathrm{~A}-\mathrm{C})$ and $2 \mathrm{~V}(\mathrm{D}-\mathrm{F})$ which were submitted to 3 cycles. A) and D) Projections taken at different tilt angles from the tilt series used to calculate the reconstructed volume. B) and E) Transversal and longitudinal ortho-slices extracted at various depths and orientations through the reconstructed volume illustrating that the SEI layer is not fully covering the SiNW structure. C) and F) 3D model of the analyzed NWs with the NW in red and the SEI layer in blue. The green arrows indicate the presence of the void between the two components of the sample.

Notably, a simple visualization of the $2 \mathrm{D}$ projections from the tilt series recorded between $\pm 70^{\circ}$ (see Figure 4A) already allows one to point out to a SEI thickness variation from the center of the NW to its borders. This observation is in agreement with our previous finding, deduced for the first type of NW (delithiation cut-off voltage of $0.6 \mathrm{~V}$ ), that the SEI is not fully 
covering the NW structure. The analysis of some typical slices taken at different depths and orientations through the corresponding reconstructed volume confirm this behavior. As highlighted in Figure 4B and E, the presence of void gaps is well present for the SiNW delithiated at a cut-off voltage of $0.8 \mathrm{~V}$, whilst no such voids could be identified for the SiNW delithiated at a cut-off voltage of $2 \mathrm{~V}$. The 3D model shown in Figure 4E depicts also the notable absence of the void gaps between the SEI layer and the SiNW delithiated at $2 \mathrm{~V}$. For these samples, a quantitative determination of the total volume of the SEI layer has also been performed using the same tomography analysis. According to the results, a relative volume of $4.732 * 10^{6} \mathrm{~nm}^{3}$ for the sample delithiated at $0.8 \mathrm{~V}$ and $11.484 * 10^{6} \mathrm{~nm}^{3}$ for the sample cycled at $2 \mathrm{~V}$ was determined. Moreover by counting the pixels located at the interface of the SEI layer and the SiNW surface we were able to estimate a total surface contact of $0.0793 * 10^{6} \mathrm{~nm}^{2}$ for the SiNW delithiated at $0.8 \mathrm{~V}$ and $0.163^{*} 10^{6} \mathrm{~nm}^{2}$ for the $\mathrm{SiNW}$ delithiated at $2 \mathrm{~V}$ respectively.

\subsubsection{Discussions on the SEI formation mechanism}

The analysis and comparison of these first series of tomographic data highlights the general trend of an inhomogeneous distribution of the as-formed SEI layer around the NWs structure and its preferential localization on opposed sides of the NW. Furthermore, stark differences can be identified when the SiNWs delithiation cut-off voltage is modified. The first one that can easily be observed from a simple visualization of the 3D models, points out an important increase of the SEI layer roughness which is clearly higher for the sample delithiated up to 2 V. The SEI layer appears to form from small agglomerates whose presence is clearly less distinguishable when the delithiation voltage is lowered. Another marked difference, easily distinguishable in Figures 3 and 4 A) - C), highlights the occurrence of a void gap between the as-formed SEI layer and the underlying NW. This gap that is undoubtedly visible for samples delithiated up to $0.8 \mathrm{~V}$ and $0.6 \mathrm{~V}$, cannot be evidenced on the sample delithiated up to $2 \mathrm{~V}$. Based on the information obtained from the ET studies, we propose in Figure 5 a schematic 
model for the geometrical characteristic of the SEI layer for each of the considered samples. The observation of such marked morphological features can be associated to the important volume changes of the NWs during the electrochemical cycling which makes difficult the formation of a morphologically stable and uniformly distributed SEI layer. As the SiNW undergoes outwards expansion, i.e. towards the electrolyte, upon lithiation and inwards contraction during délithiation, accounting for the results obtained by ET, we can reasonable presume that the SEI layer formed in the lithiated (expanded) state may easily undergo some micro-cracking during the delithiation state when the NW shrinks substantially see images Supporting Information part S3). Through the next electrochemical cycle, i.e., at micro-cracks level, the fresh electrolyte-exposed SiNW surface would undergo fresh SEI formation, resulting in a thicker and thicker SEI layer upon subsequent charge/discharge cycling (see images $\mathrm{G}$ )-H) Supporting information part S2). This sequential behavior can explain the 3D distribution of the SEI layer on the SiNW which we determined from the analysis of the reconstructed volumes. At this point, we cannot totally exclude the possibility that the sample preparation, particularly the rinsing of the electrode with DMC, may induce a partial dissolution of some local parts of the as-formed SEI layer inside the electrochemical cell. Nevertheless, such event is expected to be local and incidental whilst through all our observations, the SEI layer appeared firmly attached to the underlying NW, mainly through two contact points located on both sides of the NW, making a significant part of the underlying NW' surface less protected. In these areas, we may reasonably assume that the SEI layer is less protective and does not acts as a perfect surface passivation layer. Consequently, during the next cycle to low potentials, the electrolyte can be in direct contact with the NW' Si core surface which triggers its degradation through fresh SEI formation. When further cycling is carried, this mechanism leads to an irregular accumulation of the SEI around the NWs, as could be evidenced by the analysis of a sample that had undergone 20 cycles (see Supporting Information part Figure S2). It is 
important to note that these observations are in good agreement with the previously reported SEM characterization of cycled SiNW electrodes that underline an important accumulation of the SEI on the electrode upon cycling. Our observations revealed that the low delithiation cutoff voltages (i.e. $0.6 \mathrm{~V}$ and $0.8 \mathrm{~V}$ ) tend to induce the formation of void gaps between the SiNW and the as-formed SEI layer with the latter exhibiting irregular thickness. Inherently such void gaps trigger further core SiNWs degradation through fresh SEI formation and accumulation upon cycling and may explain the generally reported capacity fading of SiNWs electrodes. A potential route to mitigate such degradation mechanism appears in using a higher delithiation cut-off voltage of (i.e. $2 \mathrm{~V}$ ) in accordance with the previously reported studies. ${ }^{47}$ Other potential mitigation paths could be the use of smaller volume SiNPs that hold potential for better accommodation of the morphological changes induced upon cycling. To this respect a binders/additives free, hybrid SiNPs@VACNTs architecture, appears as one of the most suitable morphology. ${ }^{14,41}$

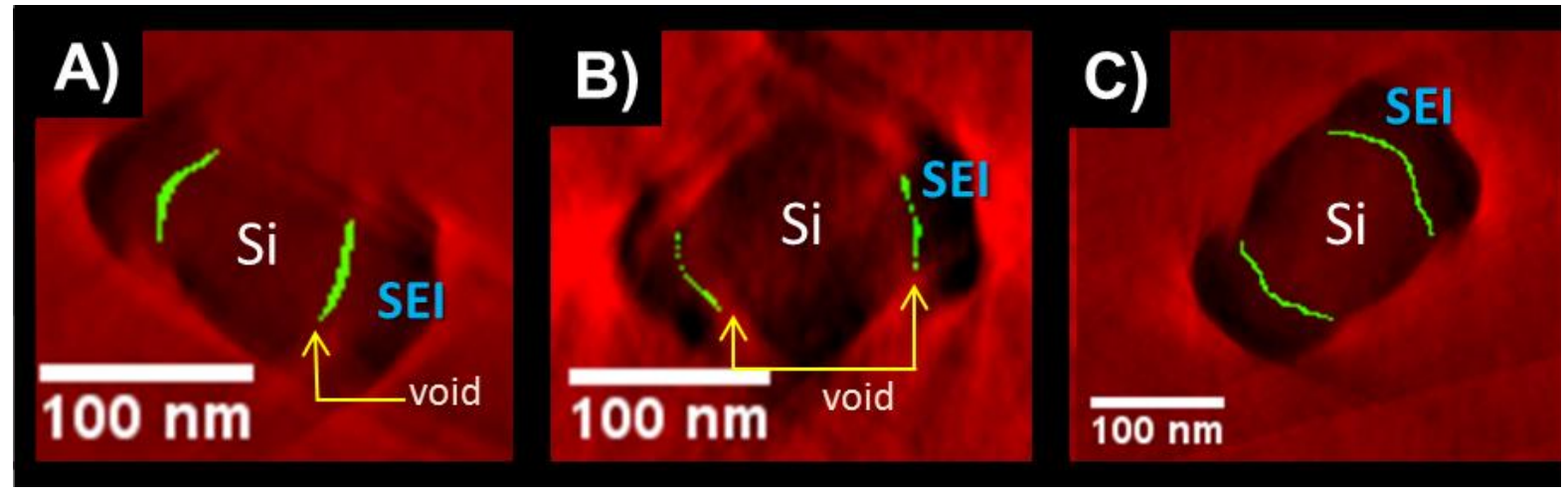

Figure 5. Cross-sectional representations of the reconstructed volume and of the 3D models, deduced from the 3D-TEM analysis, evidencing the contact surface between the as-formed SEI layer and the underlying SiNW for each studied specimen, cycled using a delithiation cut-off voltage of A) $0.6 \mathrm{~V}, \mathrm{~B}$ ) $0.8 \mathrm{~V}$ and C) $2 \mathrm{~V}$. Scale bare in the cross-section is $100 \mathrm{~nm}$.

\subsection{SiNPs@VACNTs anode material}


Using similar TEM-based characterization approach used for the SiNWs system we have investigated the morphology evolution and the 3D architecture of the second system, the SiNPs@VACNTs hierarchical hybrid nanostructure.

\subsubsection{General characteristics of the SiNPs@CNTs hybrid anode material}

The structure of the SiNPs@VACNTs hybrid nanostructure before and after electrochemical cycling was firstly characterized by classical TEM (see Figure 6 A and B). Marked morphology change of the two systems is clearly evidenced. Homogeneous SiNPs deposition on the CNTs sidewalls is highlighted in the TEM micrograph in Figure 6A( see also the SEM micrographs in figure S6 in Supporting Information). The as-synthesized SiNPs exhibit a rather spherical shape with a homogenous size distribution of $15 \pm 2.5 \mathrm{~nm}$. The highresolution TEM (HR-TEM) micrograph (inset in Figure 6A) indicates the typical amorphous structure of the SiNPs. Energy dispersive EDS line-scan chemical analyses showed in Supporting Information, Figure S4, reveals the chemical composition of the SiNPs. The NPs are mainly composed of elemental Si enclosed by a thin O rich layer $(2 \mathrm{~nm})$ at the surface which most probably appeared due to ambient exposure during the transfer from the CVD reactor to the microscope chamber.

Subsequent to the electrochemical cycling of the SiNPs@VACNTs electrode, the CNTs appear completely covered with SiNPs, leading to a rougher external surface indicative of the presence of an SEI layer, as indicated by the TEM micrograph in Figure 6B. 

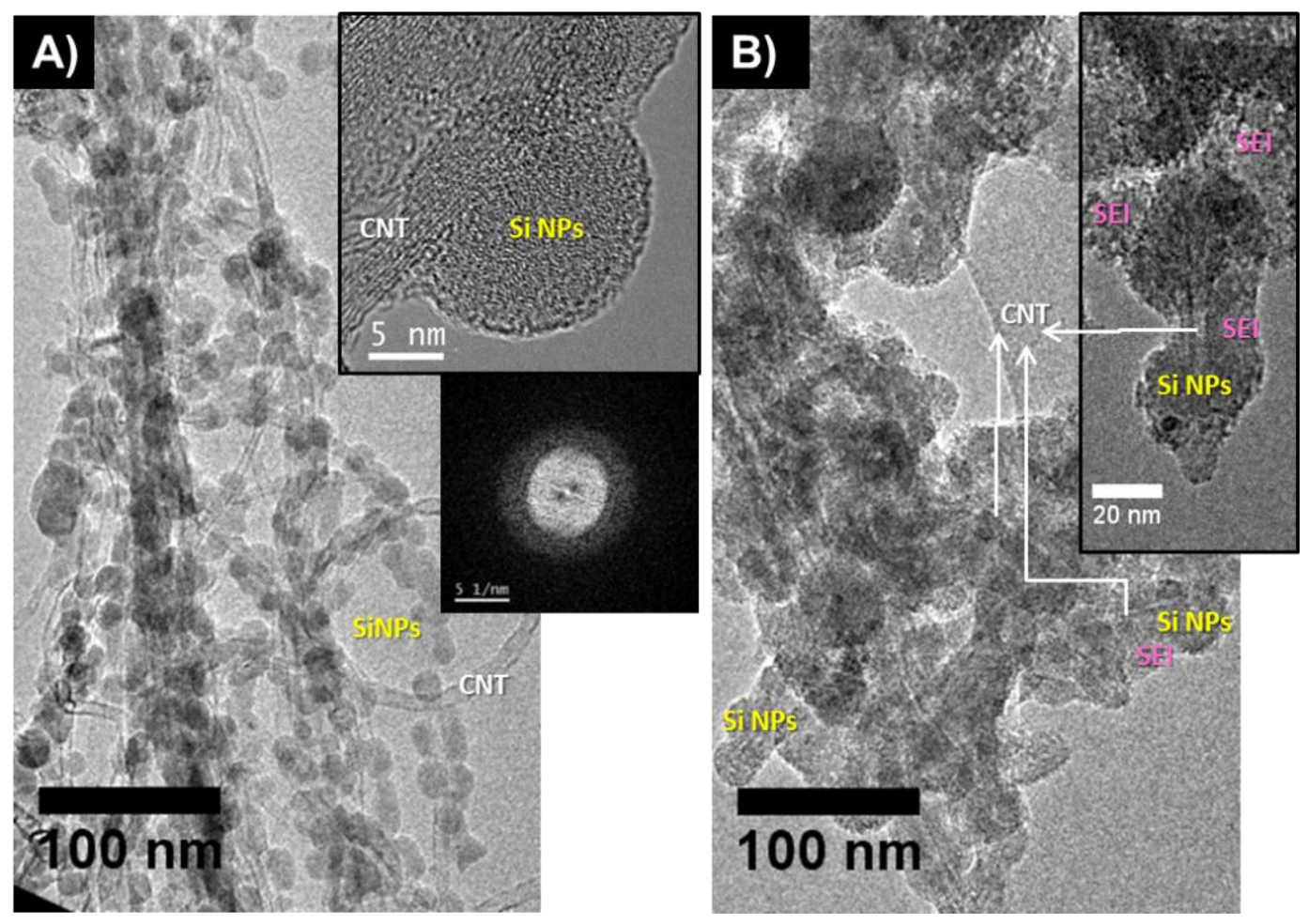

Figure 6. Typical TEM images of SiNPs@VACNTs before (A) and after (B) electrochemical cycling. Inset in (A) shows a high-resolution TEM image of a SiNP and its corresponding FFT which strongly suggest the presence of an amorphous structure. Inset in (B) shows a magnified TEM image of SiNPs@VACNTs illustrating their new morphology after cycling.

ET studies performed in the conventional TEM mode provided once again a complete 3D characterization of these nanostructures. A closer investigation of the general structure, reveals notable information in particular on the homogeneity of the CNTs decoration and the NPs anchoring characteristics with respect to the outer shell of the CNTs support. For example, we were able to verify whether there is a preferential position of SiNP anchoring with respect to the CNT support. Figure 7A presents one of the typical 2D-TEM projections from the tilt series used to reconstruct the volume of a SiNPs@VACNTs assembly obtained after 5 minutes of exposure to the $\mathrm{SiH}_{4}$ precursor; where the roughly spherical morphology of the SiNPs can be recognized. The homogeneous distribution of the SiNPs along the nanotube sidewalls is confirmed through the analysis of representative slices taken at different depths and orientations through the reconstructed volume. Remarkably, by individually analyzing several CNTs present in the reconstructed volume, we can clearly evidence that the attachment of the NPs to 
the tube occurs only at some specific locations on the surface, via the so-called anchoring points (see Figure 7C).
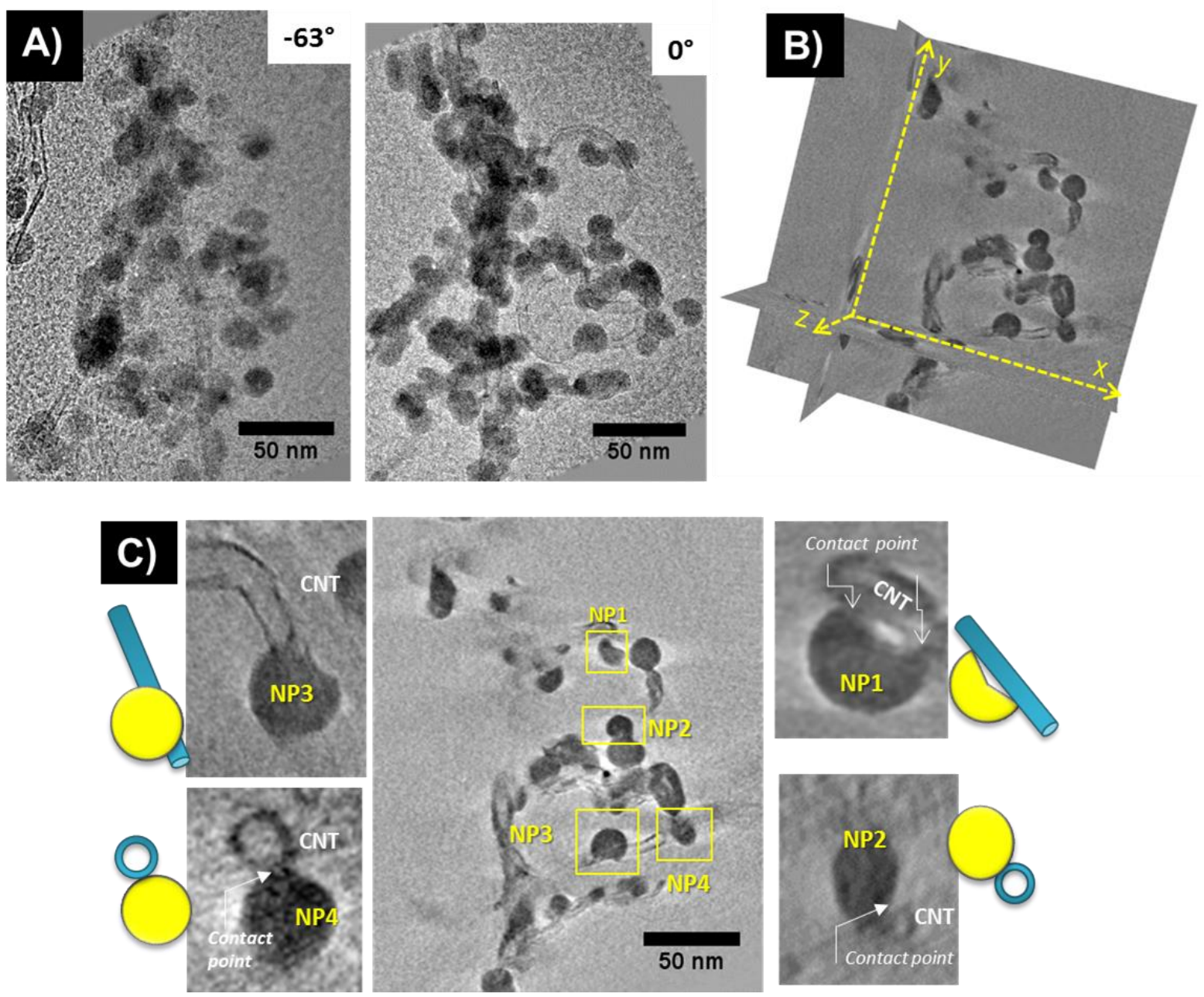

Figure 7. 3D reconstruction of a typical SiNPs@VACNTs obtained after 5 minutes of exposure of the CNTs to the $\mathrm{SiH}_{4}$ precursor. (A) 2D-TEM projections extracted from the tilt series used to calculate the volume, (B) top: typical ortho-slices extracted from the reconstructed volume, (C) zoom on four SiNPs from the reconstructed volume of the analyzed assembly evidencing their attachment to the tube sidewall via the anchoring points.

The presence of such localized anchoring points suggests the presence of some structural defects in the graphitic structure of the tubes' outer walls. Such local defects exhibit a highly increased reactivity of the tube surface towards $\mathrm{SiH}_{4}$ molecules and thus provide preferential nucleation sites for the $\mathrm{Si}$ seeds as compared to a defect free nanotubes surface. This mechanism is consistent with further ET studies performed for SiNPs@VACNTs nanostructures obtained by 10 and 15 minutes exposure to $\mathrm{SiH}_{4}$, which are summarized in 
Supporting Information part Figure S5 and Figure S6. The detailed analyses of the corresponding reconstructions reveal that the size of the as-deposited SiNPs continuously increase upon the increase of the gas exposure time and the morphology evolves towards a full nanotubes' side walls coverage for large deposition time . For 10 minutes exposure time, larger SiNPs with diameters up to $25 \pm 2.5 \mathrm{~nm}$ are observed to be attached to the CNTs side walls. However, as illustrated in Figure S5 in Supporting Information, in this case the attachment of the SiNPs evolved from local anchoring points towards larger anchoring area covering their whole underlying surface. Along with the NPs volume increase, such morphology obviously reduces the overall specific surface of the as-deposited active Si material. For a larger deposition time, we observed further lateral growth of the NPs along the nanotubes' walls which coupled with further inter-particles Si deposition, gradually lead to full nanotubes coverage with a Si thin film. The 3D reconstruction of a sample obtained after 15 minutes of exposure to $\mathrm{SiH}_{4}$ (see Figure S6 in Supporting Information) illustrates that the full external surface of the nanotube is now covered by a Si layer with a homogeneous thickness of $30 \mathrm{~nm}$, the resulting hybrid structure having an external morphology similar to a SiNW. In addition, in this last case a detailed analysis of the reconstructed volume along the tube axis demonstrates that the Si layer is tightly bonded to the tube external surface, with no interfacial gaps being observed. In this case, the specific surface of the Si active material is furthermore reduced compared to the previous two samples and it approaches that of a SiNW of equivalent diameter.

\subsubsection{D-TEM Morphology of the cycled SiNPs@VACNTs anode}

Accounting for the ET studies presented above and our previous reported studies, we focused the 3D-TEM morphology study of the SiNPs@VACNTs system on the electrodes obtained upon 5 minutes of exposure to a $\mathrm{SiH}_{4}$ and subsequent electrochemical cycling with $0.020 \mathrm{~V}$ cut-off voltage (please see also cycling data available in supporting information in Figure S8). 
In this regard, and in order to facilitate the SEI layer observation, we submitted the assynthesized SiNPs@VACNTs to one full lithiation-delithiation. For the 2D and 3D-TEM analyses several SiNPs@VACNTs assemblies were considered. Figure 8A presents three different projections extracted from one of the tilt series acquired in TEM using a very low electron dose in order to avoid the degradation of the SEI layer. As readily observed, the carbon nanotube shape and the spherical morphology of the attached SiNPs are still well discernable in some of the projections and appear to be fully covered by a thin SEI layer whose precise thickness is difficult to quantify due to the low contrast between the SiNPs and the SEI.
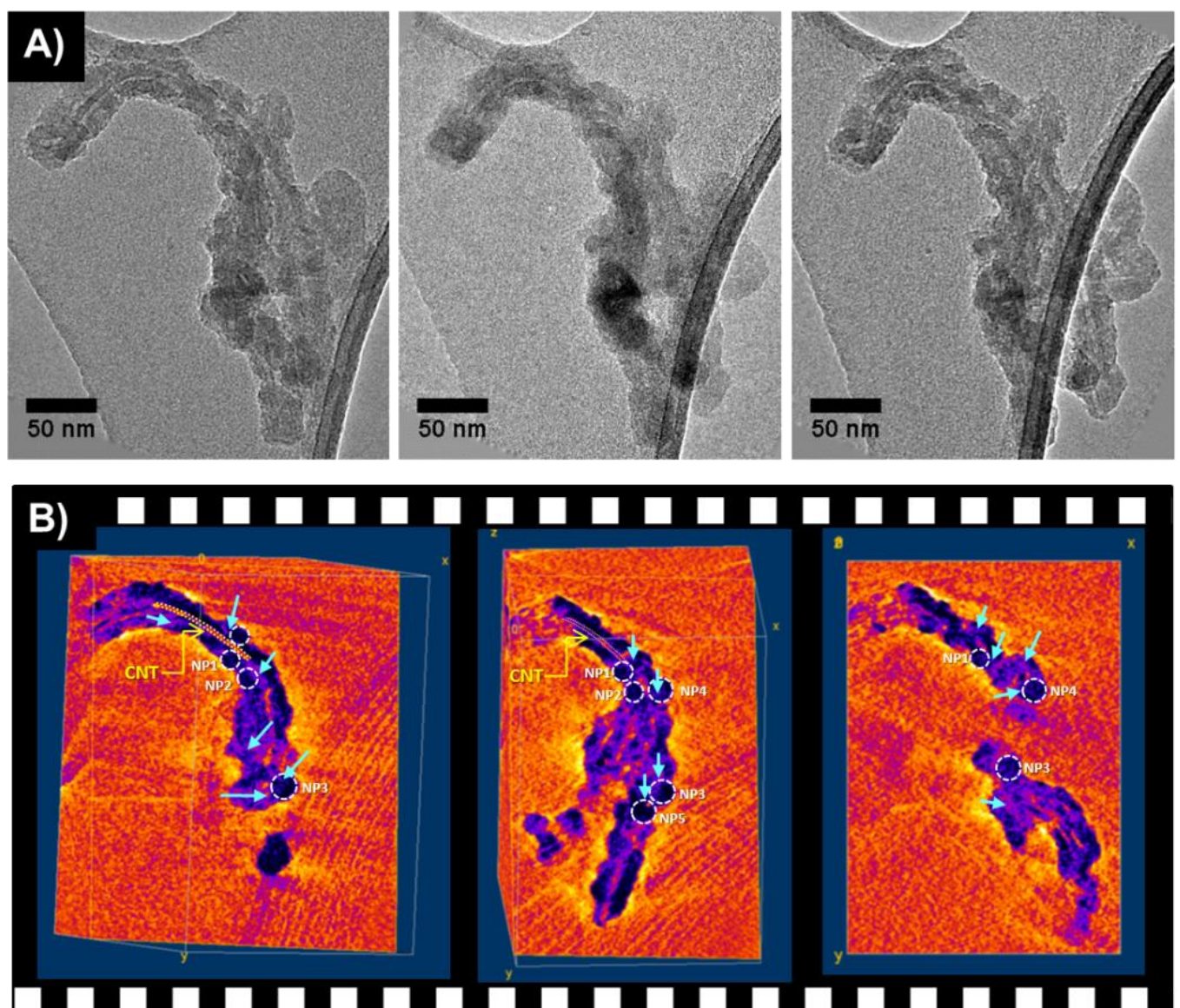

Figure 8.3D reconstruction of cycled SiNPs@VACNT. (A) 2D-TEM projections at three tilt angles extracted from the tilt series used to calculate the reconstruction, (B) sequence of three typical orthoslices extracted from the reconstructed volume.

Further investigations on the evolution of the SEI thickness could be inferred from analysis of the reconstructed volumes. Figure $8 \mathrm{~B}$ depicts a sequence of several sections extracted from 
the reconstructed volume in which the supporting nanotube is marked by white arrows. The edges of some chosen SiNPs, appearing with dark contrast in the sections, are marked by fine white lines for highlighting their respective surface contour, while the as-formed SEI layer is noted by light blue color. The as-formed SEI layer is observed to be present all around the anode material surrounding not only the SiNPs but also the space between the Si nanoparticles and has an inhomogeneous thickness. According to the investigation of the impact of SiNPs deposition time discussed above, this observation suggests that although for 5 minutes $\mathrm{SiH}_{4}$ exposure time, generally SiNPs preferentially nucleated and grown on the nanotubes side-walls, some silicon deposition (at much lower rate) may occurs on the inter-particles nanotube surface. Such inhomogeneous initial thickness may lead to an inhomogeneous density distribution of formed SEI. However, the thickness of the Si layer deposited on the inter-nanoparticles nanotube surface appear as extremely thin as evidenced from Figure 7 and cannot fully explain the observed morphology of the as-formed SEI layer. We can thus further consider that during the lithiation step, the SiNPs are outwards expanding, i.e. towards the electrolyte, allowing for the decomposition of the electrolyte with subsequent formation and growing of the SEI layer all around the SiNP surface. As the lithiation pursue, the layer thickness increases and covers all NPs while partly filling also the space between the NPs. Importantly, as the SiNPs contract during the delithiation step, the as-formed SEI layer appear to remain firmly attached to the SiNPs surface, acting as a perfect passivation layer. Such a behavior is in contrast with the observed evolution for the pure SiNWs and suggests a promising approach to improve the cycling lifetime of Si anodes when used in the form of hybrid SiNPs@VACNT nanostructures.

\section{Conclusions}

A comprehensive 3D tomographic analysis was performed on two types of binders and additives free Si-based anode materials, i.e.SiNWs and SiNPs@VACNTs, subsequent to their 
electrochemical cycling in the presence of an electrolyte containing FEC as additive. The approach allowed retrieving structural information about the nano-Si material morphological evolution and the corresponding SEI layer formation and its distribution upon the samples cycling. The detailed analysis of the reconstructed volume corresponding to each studied SiNW highlighted the occurrence of inhomogeneous distribution of the as-formed SEI layer around the underlying SiNW. The as-formed SEI layer exhibits preferential localization on opposite sides of the SiNW and different morphologies depending upon the cut-off delithiation voltages $(0.6 \mathrm{~V}, 0.8 \mathrm{~V}$ and $2 \mathrm{~V})$. The 3D model clearly indicates that the roughness of the SEI layer is more important for the sample delithiated up to $2 \mathrm{~V}$. In contrast with the sample delithiated to $2 \mathrm{~V}$, ET studies highlighted the occurrence of voids between the SEI layer and the NW for the samples delithiated down to $0.8 \mathrm{~V}$ and $0.6 \mathrm{~V}$, thus leaving exposed part of the Si core in contact with the electrolyte. Inherently, such void gaps can trigger an increase in the SiNWs' core degradation through fresh SEI formation and accumulation upon further cycling and may explain the generally reported capacity fading of SiNWs electrodes. Potential routes to mitigate such degradation mechanism appear to be the use of a higher délithiation cut-off voltage of (i.e. $2 \mathrm{~V}$ ) in accordance with the previously reported studies and/or the use of smaller volume SiNPs as hybrid SiNPs@VACNTs architecture.

ET analysis of the SiNPs@VACNT hybrid heterostructures, provided direct information on the distribution and the localization of the SiNPs at the surface of the CNTs skeleton. We now are able to see that the as-grown NPs appear to preferentially nucleate and grow attached to the nanotubes' graphitic planes via some "contact points" whose localization can be assigned to the existence of local defects within the graphitic structure. As revealed by the ET studies for increasing deposition time, the resulting structure undergo continuous morphology change from discreet nanoparticles attached to CNTs walls up to full external surface coverage of the nanotube by a Si layer with homogeneous thickness. Thus the resulting hybrid structure mimics 
an external morphology similar to a SiNW system. Analysis performed subsequent to electrochemical cycling of the SiNPs@VACNT specimens nanostructures, revealed that the asformed SEI remain firmly attached to the underlying SiNPs' surface. Such configuration potentially can alleviate the void gaps triggered degradation mechanism observed for the pure SiNWs structures which led us to conclude that the SiNPs morphology is probably much more suitable to be considered as active material for $\mathrm{LiB}$ anodes with improved cycling life-time.

We believe that these results provide valuable information for the electrochemical community. Further experiments using liquid cells inside a TEM coupled with spectroscopy techniques are needed to observe the dynamics of $\mathrm{Li}^{+}$-ion insertion and extraction and thus the real-time degradation mechanism of the SEI layer.

\section{Supporting Information}

Movie S1: Projection tilt series corresponding to the SiNW cycled using a delithiation cut-off voltage of $0.6 \mathrm{~V}$ and that suffered 3 cycles;

Movie S2: 3D-Model of the SiNW cycled using a delithiation cut-off voltage of $0.6 \mathrm{~V}$ and that suffered 3 cycles;

Movie S3: Projection tilt series corresponding to the SiNW cycled using a delithiation cut-off voltage of $0.8 \mathrm{~V}$ and that suffered 3 cycles;

Movie S4: 3D-Model of the SiNW cycled using a delithiation cut-off voltage of $0.8 \mathrm{~V}$ and that suffered 3 cycles;

Movie S5: Projection tilt series corresponding to the SiNW cycled using a delithiation cut-off voltage of $2 \mathrm{~V}$ and that suffered 3 cycles;

Movie S6: 3D-Model of the SiNW cycled using a delithiation cut-off voltage of $2 \mathrm{~V}$ and that suffered 3 cycles;

\section{Acknowledgements}

The authors acknowledge financial support from Chaire EDF "Energies Durable", the French state managed by the National Research Agency under the Investments for the Future program under the references ANR-10-EQPX-50 pole NanoMax \& \&NanoTEM. For the tomography studies, the authors acknowledge financial support from the CNRS and CEA METSA electron microscopy network (FR3507). IF and LL would like to thank Sergio Marco for the Cryogenic sample holder used for the 2D-TEM preliminary experiments. The authors would also like to 
acknowledge the Centre Interdisciplinaire de Microscopie électronique de l'X (CIMEX). This work is part of the NanoMaDe-3E Initiative.

\section{References:}

(1) Tarascon, J.-M.; Armand, M. Issues and Challenges Facing Rechargeable Lithium Batteries. Nature 2001, 414, 359-367.

(2) Goodenough, J.-B.; Kim, Y. Challenges for Rechargeable Li Batteries. Chem. Mater 2010, 22, 587-603.

(3) Etacheri, V.; Marom, R.; Elazari, R.; Salitra, G.; Aurbach, D. Challenges in the Development of Advanced Li-Ion Batteries: A Review. Energy Environ. Sci. 2011, 4, 3243-3262.

(4) Guerard, D.; Herold, A. Intercalation of Lithium into Graphite and Other Carbons. Carbon N. Y. 1975, 13, 337-345.

(5) Wang, C. S.; Wu, G. T.; Li, W. Z. Lithium Insertion in Ball-Milled Graphite. J. Power Sources 1998, 76, 1-10.

(6) Nitta, N.; Wu, F.; Lee, J. T.; Yushin, G. Li-Ion Battery Materials: Present and Future. Mater. Today 2015, 18, 252-264.

(7) Obrovac, M. N.; Krause, L. J. Reversible Cycling of Crystalline Silicon Powder. J. Electrochem. Soc. 2007, 154, A103-A108.

(8) Liu, X. H.; Zheng, H.; Zhong, L.; Huang, S.; Karki, K.; Zhang, L. Q.; Liu, Y.; Kushima, A.; Liang, W. T.; Wang, J. W.; Cho, J. H.; Epstein, E.; Dayeh, S. A.; Picraux, S. T.; Zhu, T.; Li, J.; Sullivan, J. P.; Cumings, J.; Wang, C.; Mao, S. X.; Ye, Z. Z.; Zhang, S.; Huang, J. Y. Anisotropic Swelling and Fracture of Silicon Nanowires during Lithiation. Nano Lett. 2011, 11 (8), 3312-3318. https://doi.org/10.1021/nl201684d.

(9) Jin, Y.; Zhu, B.; Lu, Z.; Liu, N.; Zhu, J. Challenges and Recent Progress in the Development of Si Anodes for Lithium-Ion Battery. Adv. Energy Mater. 2017, 7 (23), 1-17. https://doi.org/10.1002/aenm.201700715.

(10) Takamura, T.; Ohara, S.; Uehara, M.; Suzuki, J.; Sekine, K. A Vacuum Deposited Si Film Having a Li Extraction Capacity over $2000 \mathrm{MAh} / \mathrm{g}$ with a Long Cycle Life. J. Power Sources 2004, 129, 96-100.

(11) Ulldemolins, M.; LeCras, F.; Pecquenard, B.; Phan, V. P.; Martin, L.; Martinez, H. Investigation on the Part Played by the Solid Electrolyte Interphase on the Electrochemical Performances of the Silicon Electrode for Lithium-Ion Batteries. $J$. Power Sources 2012, 206, 245-252.

(12) Wang, W.; Kumta, P.-N. Nanostructured Hybrid Silicon/Carbon Nanotube Heterostructures: Reversible High-Capacity Lithium-Ion Anodes. ACS Nano 2010, 4, 2233-2241.

(13) Wang, W.; Epur, R.; Kumta, P.-N. Vertically Aligned Silicon/Carbon Nanotube (VASCNT) Arrays: Hierarchical Anodes for Lithium-Ion Battery. Electrochem. Commun. 2011, 13, 429-432.

(14) Gohier, A.; Laik, B.; Kim, K.-H.; Maurice, J.-L.; Pereira-Ramos, J.-P.; Cojocaru, C. S.; Van, P. T. High-Rate Capability Silicon Decorated Vertically Aligned Carbon Nanotubes for Li-Ion Batteries. Adv. Mater. 2012, 24, 2592-2597.

(15) Epur, R.; Ramanathan, M.; Datta, M. K.; Hong, D. H.; Jampani, P. H.; Gattu, B.; Kumta, P.-N. Scribable Multi-Walled Carbon Nanotube-Silicon Nanocomposite: A Viable Lithium-Ion Battery System. Nanoscale 2015, 7, 3504-3510.

(16) Chen, Y.; Du, N.; Zhang, H.; Yang, D. Facile Synthesis of Uniform MWCNT@Si Nanocomposites as High-Performance Anode Materials for Lithium-Ion Batteries. $J$. 
Alloy. Compd. 2015, 622, 966-972.

(17) Zhong, L.; Guo, J.; Mangolini, L. A Stable Silicon Anode Based on the Uniform Dispersion of Quantum Dots in a Polymer Matrix. J. Power Sources 2015, 273, 638644.

(18) Andersen, H. F.; Foss, C. E. L.; Voje, J.; Tronstad, R.; Mokkelbost, T.; Vullum, P. E.; Ulvestad, A.; Kirkengen, M.; Mæhlen, J. P. Silicon-Carbon Composite Anodes from Industrial Battery Grade Silicon. Sci. Rep. 2019, 9 (1), 1-9. https://doi.org/10.1038/s41598-019-51324-4.

(19) Zheng, Y.; Seifert, H. J.; Shi, H.; Zhang, Y.; Kübel, C.; Pfleging, W. 3D Silicon/Graphite Composite Electrodes for High-Energy Lithium-Ion Batteries. Electrochim. Acta 2019, 317, 502-508. https://doi.org/10.1016/j.electacta.2019.05.064.

(20) Kim, H.; Han, B.; Choo, J.; Cho, J. Three-Dimensional Porous Silicon Particles for Use in High-Performance Lithium Secondary Batteries. Angew. Chemie - Int. Ed. 2008, 47 (52), 10151-10154. https://doi.org/10.1002/anie.200804355.

(21) Qu, Y.; Liao, L.; Li, Y.; Zhang, H.; Huang, Y.; Duan, X. Electrically Conductive and Optically Active Porous Silicon Nanowires. Nano Lett. 2009, 9 (12), 4539-4543. https://doi.org/10.1021/n1903030h.

(22) Ge, M.; Rong, J.; Fang, X.; Zhang, A.; Lu, Y.; Zhou, C. Scalable Preparation of Porous Silicon Nanoparticles and Their Application for Lithium-Ion Battery Anodes. Nano Res. 2013, 6 (3), 174-181. https://doi.org/10.1007/s12274-013-0293-y.

(23) Ge, M.; Rong, J.; Fang, X.; Zhou, C. Porous Doped Silicon Nanowires for Lithium Ion Battery Anode with Long Cycle Life. 2012. https://doi.org/10.1021/nl300206e.

(24) Rahman, M. A.; Song, G.; Bhatt, A. I.; Wong, Y. C.; Wen, C. Nanostructured Silicon Anodes for High-Performance Lithium-Ion Batteries. Adv. Funct. Mater. 2016, 26 (5), 647-678. https://doi.org/10.1002/adfm.201502959.

(25) Yao, Y.; McDowell, M. T.; Ryu, I.; Wu, H.; Liu, N.; Hu, L.; Nix, W. D.; Cui, Y. Interconnected Silicon Hollow Nanospheres for Lithium-Ion Battery Anodes with Long Cycle Life. Nano Lett. 2011, 11 (7), 2949-2954. https://doi.org/10.1021/nl201470j.

(26) Zhao, Y.; Liu, X.; Li, H.; Zhai, T.; Zhou, H. Hierarchical Micro/Nano Porous Silicon Li-Ion Battery Anodes. Chem. Commun. 2012, 48 (42), 5079-5081. https://doi.org/10.1039/c2cc31476b.

(27) Gowda, S. R.; Pushparaj, V.; Herle, S.; Girishkumar, G.; Gordon, J. G.; Gullapalli, H.; Zhan, X.; Ajayan, P. M.; Reddy, A. L. M. Three-Dimensionally Engineered Porous Silicon Electrodes for Li Ion Batteries. Nano Lett. 2012, 12 (12), 6060-6065. https://doi.org/10.1021/nl302114j.

(28) Chan, C.-K.; Peng, H.; Liu, G.; McIlwrath, K.; Zhang, X.-F.; Huggins, R.-A.; Cui, Y. High-Performance Lithium Battery Anodes Using Silicon Nanowires. Nat. Nanotech. 2008, 3, 31-35.

(29) Peng, K.; Jie, J.; Zhang, W.; Lee, S.-T. Silicon Nanowires for Rechargeable LithiumIon Batteries Anodes.. Appl. Phys. Lett. 2008, 93, 033105.

(30) Teki, R.; Datta, M.-K.; Krishnan, R.; Parker, T.-C.; Lu, T.-M.; Kumta, P.-N.; Koratkar, N. Nanostructured Silicon Anodes for Lithium Ion Rechargeable Batteries. Small 2009, 5, 2236-2242.

(31) Cui, L.-F.; Ruffo, R.; Chan, C.-K.; Peng, H.; Cui, Y. Crystalline-Amorphous CoreShell Silicon Nanowires for High Capacity and High Current Battery Electrodes. Nano Lett. 2009, 9, 491-495.

(32) Chan, C. K.; Patel, R. N.; O’Connell, M. J.; Korgel, B. A.; Cui, Y. Solution-Grown Silicon Nanowires for Lithium-Ion Battery Anodes. ACS Nano 2010, 4 (3), 1443-1450. https://doi.org/10.1021/nn901409q. 
(33) Zhu, B.; Jin, Y.; Tan, Y.; Zong, L.; Hu, Y.; Chen, L.; Chen, Y.; Zhang, Q.; Zhu, J. Scalable Production of Si Nanoparticles Directly from Low Grade Sources for Lithium-Ion Battery Anode. Nano Lett. 2015, 15, 5750-5754.

(34) Choi, H. S.; Kim, S. J.; Choi, H. W.; Park, C.-E.; Gao, Y. J.; Hang, Y.; Jeong, S.-Y.; Kim, J.-P.; Bae, J.-S.; Cho, C.-R. Enhanced Cycle Stability of Silicon Nanoparticles Coated with Nitrogen-Doped Carbon Layer for Lithium-Ion Battery Anode. Curr. Appl. Phys. 2017, 17, 1087-1093.

(35) Thakur, M.; Sinsabaugh, S. L.; Isaacson, M. J.; Wong, M. S.; Biswal, S. L. Inexpensive Method for Producing Macroporous Silicon Particulates (MPSPs) with Pyrolyzed Polyacrylonitrile for Lithium Ion Batteries. Sci. Rep. 2012, 2, 1-7. https://doi.org/10.1038/srep00795.

(36) Wu, H.; Zheng, G.; Liu, N.; Carney, T.-J.; Yang, Y.; Cui, Y. Engineering Empty Space between Si Nanoparticles for Lithium-Ion Battery Anodes. Nano Lett. 2012, 12, 904 909.

(37) Bang, B. M.; Kim, H.; Song, H. K.; Cho, J.; Park, S. Scalable Approach to MultiDimensional Bulk Si Anodes via Metal-Assisted Chemical Etching. Energy Environ. Sci. 2011, 4 (12), 5013-5019. https://doi.org/10.1039/c1ee02310a.

(38) Laik, B.; Eude, L.; Pereira-ramos, J. P.; Sorin, C.; Laik, B.; Eude, L.; Pereira-ramos, J. P.; Cojocaru, C. S.; Pribat, D.; La, B. Silicon Nanowires as Negative Electrode for Lithium-Ion Microbatteries Ere To Cite This Version : Electrochimica Acta Silicon Nanowires as Negative Electrode for Lithium-Ion Microbatteries. 2013.

(39) Sun, L.; Su, T.; Xu, L.; Du, H. Bin. Preparation of Uniform Si Nanoparticles for HighPerformance Li-Ion Battery Anodes. Phys. Chem. Chem. Phys. 2016, 18 (3), 15211525. https://doi.org/10.1039/c5cp06585b.

(40) Roy, P.; Srivastava, S. K. Nanostructured Anode Materials for Lithium Ion Batteries. J. Mater. Chem. A, 2015, 3, 2454-2484.

(41) Ezzedine, M. Fabrication of Hierarchical Hybrid Nanostructured Electrodes Based on Nanoparticles Decorated Carbon Nanotubes for Li-Ion Batteries To Cite This Version : HAL Id : Tel-01968037. 2017.

(42) Nawatechnologies http://www.nawatechnologies.com.

(43) Yang, Y.; Yuan, W.; Kang, W.; Ye, Y.; Pan, Q.; Zhang, X.; Ke, Y.; Wang, C.; Qiu, Z.; Tang, Y. A Review on Silicon Nanowire-Based Anodes for next-Generation HighPerformance Lithium-Ion Batteries from a Material-Based Perspective. Sustain. Energy Fuels 2020, 4 (4), 1577-1594. https://doi.org/10.1039/c9se01165j.

(44) Lei, J.; Li, L.; Kostecki, R.; Muller, R.; McLarnon, F. Characterization of SEI Layers on LiMn[Sub 2] O[Sub 4] Cathodes with In Situ Spectroscopic Ellipsometry. $J$. Electrochem. Soc. 2005, 152 (4), A774. https://doi.org/10.1149/1.1867652.

(45) Key, B.; Bhattacharyya, R.; Morcrette, M.; Seznéc, V.; Tarascon, J. M.; Grey, C. P. Real-Time NMR Investigations of Structural Changes in Silicon Electrodes for Lithium-Ion Batteries. J. Am. Chem. Soc. 2009, 131 (26), 9239-9249. https://doi.org/10.1021/ja8086278.

(46) Bhattacharyya, R.; Key, B.; Chen, H.; Best, A. S.; Hollenkamp, A. F.; Grey, C. P. In Situ NMR Observation of the Formation of Metallic Lithium Microstructures in Lithium Batteries. Nat. Mater. 2010, 9 (6), 504-510. https://doi.org/10.1038/nmat2764.

(47) Leveau, L.; Laïk, B.; Pereira-Ramos, J. P.; Gohier, A.; Tran-Van, P.; Cojocaru, C. S. Cycling Strategies for Optimizing Silicon Nanowires Performance as Negative Electrode for Lithium Battery. Electrochim. Acta 2015, 157, 218-224. https://doi.org/10.1016/j.electacta.2015.01.037.

(48) Chattopadhyay, S.; Lipson, A. L.; Karmel, H. J.; Emery, J. D.; Fister, T. T.; Fenter, P. A.; Hersam, M. C.; Bedzyk, M. J. In Situ X-Ray Study of the Solid Electrolyte 
Interphase (SEI) Formation on Graphene as a Model Li-Ion Battery Anode. Chem. Mater. 2012, 24 (15), 3038-3043. https://doi.org/10.1021/cm301584r.

(49) Itagaki, M.; Kobari, N.; Yotsuda, S.; Watanabe, K.; Kinoshita, S.; Ue, M. In Situ Electrochemical Impedance Spectroscopy to Investigate Negative Electrode of Lithium-Ion Rechargeable Batteries. J. Power Sources 2004, 135 (1-2), 255-261. https://doi.org/10.1016/j.jpowsour.2004.04.004.

(50) Lee, S. H.; You, H. G.; Han, K. S.; Kim, J.; Jung, I. H.; Song, J. H. A New Approach to Surface Properties of Solid Electrolyte Interphase on a Graphite Negative Electrode. J. Power Sources 2014, 247, 307-313. https://doi.org/10.1016/j.jpowsour.2013.08.105.

(51) Tsuda, T.; Kanetsuku, T.; Sano, T.; Oshima, Y.; Ui, K.; Yamagata, M.; Ishikawa, M.; Kuwabata, S. In Situ SEM Observation of the Si Negative Electrode Reaction in an Ionic-Liquid-Based Lithium-Ion Secondary Battery. Microscopy 2015, 64 (3), 159168. https://doi.org/10.1093/jmicro/dfv003.

(52) Liu, X. H.; Zhang, L. Q.; Zhong, L.; Liu, Y.; Zheng, H.; Wang, J. W.; Cho, J. H.; Dayeh, S. A.; Picraux, S. T.; Sullivan, J. P.; Mao, S. X.; Ye, Z. Z.; Huang, J. Y. Ultrafast Electrochemical Lithiation of Individual Si Nanowire Anodes. Nano Lett. 2011, 11 (6), 2251-2258. https://doi.org/10.1021/n1200412p.

(53) Liu, X. H.; Huang, S.; Picraux, S. T.; Li, J.; Zhu, T.; Huang, J. Y. Reversible Nanopore Formation in Ge Nanowires during Lithiation- Delithiation Cycling: An in Situ Transmission Electron Microscopy Study. Nano Lett. 2011, 11 (9), 3991-3997. https://doi.org/10.1021/nl2024118.

(54) Zhang, L. Q.; Liu, X. H.; Liu, Y.; Huang, S.; Zhu, T.; Gui, L.; Mao, S. X.; Ye, Z. Z.; Wang, C. M.; Sullivan, J. P.; Huang, J. Y. Controlling the Lithiation-Induced Strain and Charging Rate in Nanowire Electrodes by Coating. ACS Nano 2011, 5 (6), 4800 4809. https://doi.org/10.1021/nn200770p.

(55) Hou, C.; Han, J.; Liu, P.; Yang, C.; Huang, G.; Fujita, T.; Hirata, A.; Chen, M. Operando Observations of SEI Film Evolution by Mass-Sensitive Scanning Transmission Electron Microscopy. Adv. Energy Mater. 2019, 9 (45). https://doi.org/10.1002/aenm.201902675.

(56) Karakulina, O. M.; Demortière, A.; Dachraoui, W.; Abakumov, A. M.; Hadermann, J. In Situ Electron Diffraction Tomography Using a Liquid-Electrochemical Transmission Electron Microscopy Cell for Crystal Structure Determination of Cathode Materials for Li-Ion Batteries. Nano Lett. 2018, 18 (10), 6286-6291. https://doi.org/10.1021/acs.nanolett.8b02436.

(57) Lutz, L.; Dachraoui, W.; Demortière, A.; Johnson, L. R.; Bruce, P. G.; Grimaud, A.; Tarascon, J. M. Operando Monitoring of the Solution-Mediated Discharge and Charge Processes in a Na-O2 Battery Using Liquid-Electrochemical Transmission Electron Microscopy. Nano Lett. 2018, 18 (2), 1280-1289. https://doi.org/10.1021/acs.nanolett.7b04937.

(58) Ersen, O.; Florea, I.; Hirlimann, C.; Pham-Huu, C. Exploring Nanomaterials with 3D Electron Microscopy. Mater. Today 2015, 18, 395-408.

(59) Melinte, G.; Florea, I.; Moldovan, S.; Janowska, I.; Baaziz, W.; Arenal, R.; Wisnet, A.; Scheu, C.; Begin-Colin, S.; Begin, D.; Pham-Huu, C.; Ersen, O. A 3D Insight on the Catalytic Nanostructuration of Few-Layer Graphene. Nat. Commun. 2014, 5 (May), 18. https://doi.org/10.1038/ncomms5109.

(60) Florea, I.; Ihiawakrim, D.; Hirlimann, C.; Ersen, O. Three-Dimensional Tomographic Analyses of CeO 2 Nanoparticles. 2013.

(61) Koneti, S.; Borges, J.; Roiban, L.; Rodrigues, M. S.; Martin, N.; Epicier, T.; Vaz, F.; Steyer, P. Electron Tomography of Plasmonic Au Nanoparticles Dispersed in a TiO 2 Dielectric Matrix. ACS Appl. Mater. Interfaces 2018, 10 (49), 42882-42890. 
https://doi.org/10.1021/acsami.8b16436.

(62) Ge, M.; Lu, Y.; Ercius, P.; Rong, J.; Fang, X.; Mecklenburg, M.; Zhou, C. Large-Scale Fabrication, 3D Tomography, and Lithium-Ion Battery Application of Porous Silicon. Nano Lett. 2014, 14 (1), 261-268. https://doi.org/10.1021/nl403923s.

(63) Crowther RA; DeRosier DJ; Klug A. Reconstruction of a Three-Dimensional Structure From Projection and Its Application To Electron Microscopy. 1970, 317 (1530), 319340. https://doi.org/10.1098/rspa.1970.0119.

(64) Cedex, V. Fast ' Operando ' Electron Nanotomography. 2018, 269 (2), 117-126. https://doi.org/10.1111/jmi.12557.

(65) Vanrompay, H.; Bladt, E.; Albrecht, W.; Béché, A.; Zakhozheva, M.; Sánchez-Iglesias, A.; Liz-Marzán, L. M.; Bals, S. 3D Characterization of Heat-Induced Morphological Changes of Au Nanostars by Fast: In Situ Electron Tomography. Nanoscale 2018, 10 (48), 22792-22801. https://doi.org/10.1039/c8nr08376b.

(66) Kim, K.-H.; Gohier, A.; Bourée, J. E.; Chatelet, M.; C-Sorin Cojocaru. The Role of Catalytic Nanoparticle Pretreatment on the Growth of Vertically Aligned Carbon Nanotubes by Hot-Filament Chemical Vapor Deposition. Thin Solid Films 2015, 575 , 84-91.

(67) Mastronarde, D. N. Dual-Axis Tomography: An Approach with Alignment Methods That Preserve Resolution. J. Struct. Biol. 1997, 120 (3), 343-352. https://doi.org/10.1006/jsbi.1997.3919.

(68) Gordon, R.; Bender, R.; Herman, G. T. Algebraic Reconstruction Techniques (ART) for Three-Dimensional Electron Microscopy and X-Ray Photography. J. Theor. Biol. 1970, 29 (3), 471-481. https://doi.org/10.1016/0022-5193(70)90109-8.

(69) MessaoudiI, C.; Boudier, T.; Sorzano, C. O. S.; Marco, S. TomoJ: Tomography Software for Three-Dimensional Reconstruction in Transmission Electron Microscopy. BMC Bioinformatics 2007, 8, 1-9. https://doi.org/10.1186/1471-2105-8-288.

(70) Midgley, P. A.; Weyland, M. 3D Electron Microscopy in the Physical Sciences: The Development of Z-Contrast and EFTEM Tomography. Ultramicroscopy 2003, 96 (34), 413-431. https://doi.org/10.1016/S0304-3991(03)00105-0.

(71) Koster, A. J.; Ziese, U.; Verkleij, A. J.; Janssen, A. H.; De Jong, K. P. ThreeDimensional Transmission Electron Microscopy: A Novel Imaging and Characterization Technique with Nanometer Scale Resolution for Materials Science. $J$. Phys. Chem. B 2000, 104 (40), 9368-9370. https://doi.org/10.1021/jp0015628.

(72) Ersen, O.; Hirlimann, C.; Drillon, M.; Werckmann, J.; Tihay, F.; Pham-Huu, C.; Crucifix, C.; Schultz, P. 3D-TEM Characterization of Nanometric Objects. Solid State Sci. 2007, 9 (12), 1088-1098. https://doi.org/10.1016/j.solidstatesciences.2007.09.018. 


\title{
Supporting Information
}

\section{Insight into the formation and stability of solid}

\author{
electrolyte interphase (SEI) for nanostructured silicon-
}

\section{based anode electrodes used in Li-ion batteries}

\author{
Mariam EZZEDINE ${ }^{1}$, Mihai-Robert ZAMFIR ${ }^{1,2}$, Fatme JARDALI ${ }^{1}$, Lucie LEVEAU $^{1,3}$ \\ Eleonor CARISTAN ${ }^{1}$, Ovidiu ERSEN ${ }^{4}$, Costel-Sorin COJOCARU ${ }^{1}$, Ileana FLOREA ${ }^{1, \text { * }}$
}

\author{
${ }^{1}$ LPICM, CNRS, Ecole polytechnique, IPParis, 91228 Palaiseau, France \\ ${ }^{2}$ National Institute for Laser, Plasma \& Radiation Physics (INFLPR), Atomistilor Street, No. 409, \\ Magurele, Ilfov RO-077125, Romania \\ ${ }^{3}$ Renault SAS, DREAM/DETA/SEE, 1, Avenue du Golf, 78288 Guyancourt, France \\ ${ }^{4}$ Institut de Physique et Chimie des Matériaux de Strasbourg (IPCMS), UMR 7504 CNRS - Université de \\ Strasbourg, 23 rue du Loess, 67034 Strasbourg Cedex 2, France \\ *lenuta-ileana.florea@polytechnique.Edu
}

Movie S1: Projection tilt series corresponding to the SiNW cycled using a delithiation cut-off voltage of $0.6 \mathrm{~V}$ and that suffered 3 cycles;

Movie S2: 3D-Model of the SiNW cycled using a delithiation cut-off voltage of $0.6 \mathrm{~V}$ and that suffered 3 cycles;

Movie S3: Projection tilt series corresponding to the SiNW cycled using a delithiation cut-off voltage of $0.8 \mathrm{~V}$ and that suffered 3 cycles;

Movie S4: 3D-Model of the SiNW cycled using a delithiation cut-off voltage of $0.8 \mathrm{~V}$ and that suffered 3 cycles;

Movie S5: Projection tilt series corresponding to the SiNW cycled using a delithiation cut-off voltage of $2 \mathrm{~V}$ and that suffered 3 cycles;

MovieS6: 3D-Model of the SiNW cycled using a delithiation cut-off voltage of $2 \mathrm{~V}$ and that suffered 3 cycles; 
S1 : TEM images and its corresponding electron Diffraction pattern of a cycled SiNW

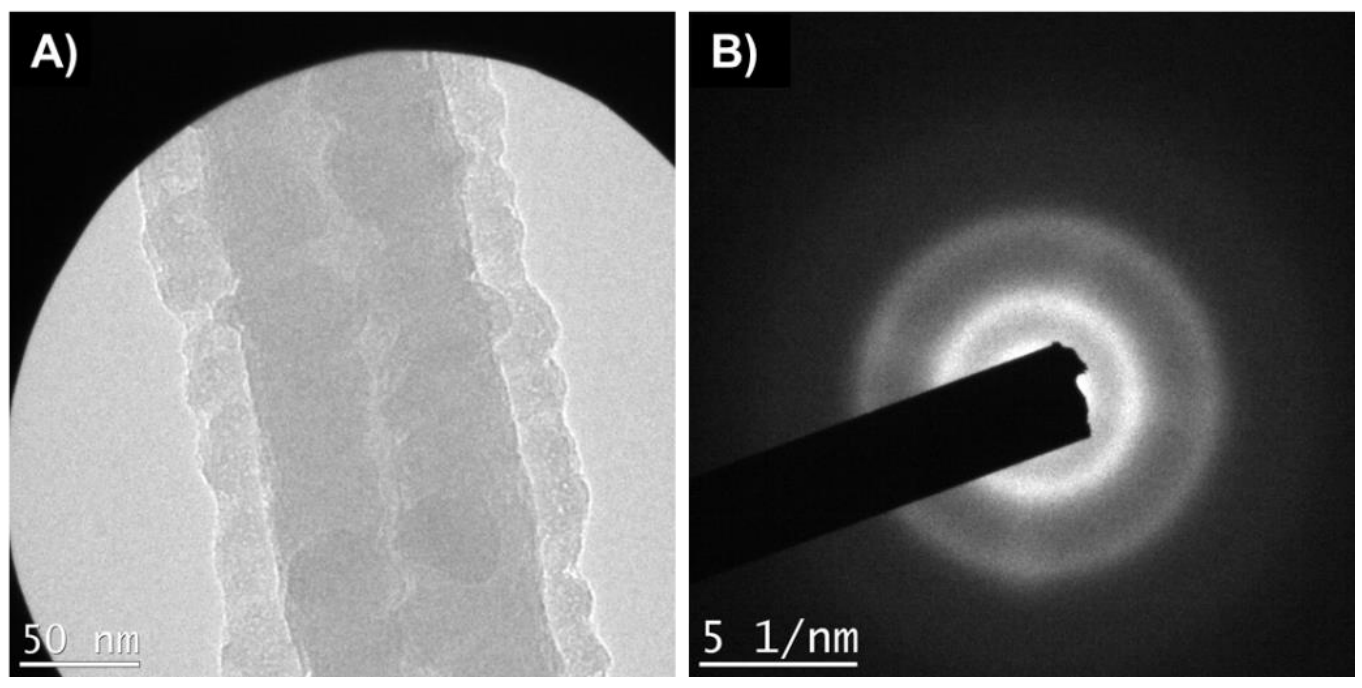


S2: 2D-TEM micrographs of different SiNWs which were cycled under different number of cycles for $0.6 \mathrm{~V}$ delithiation cut-off voltages: A)-C) 1 cycle; D)- F) 3cycles; G)J) 20 cycles

As it can be observed after first cycle the SEI follows the SiNWs pristine morphology figure A-C) fully covering the NW surface in an inhomogeneous manner and presenting a rough surface. After 3cycles the nanowire morphology can still be distinguish in the middle with SEI at its surface presenting an inhomogeneous covering. For some cycled SiNWs one can clearly see the SiNWs core (see figure D) and F). Moreover as the number of cycles increases to 20 (figure G-J) the morphology of the cycled systems dramatically changes, and the wire morphology is completely hidden by the inhomogeneous SEI covering.
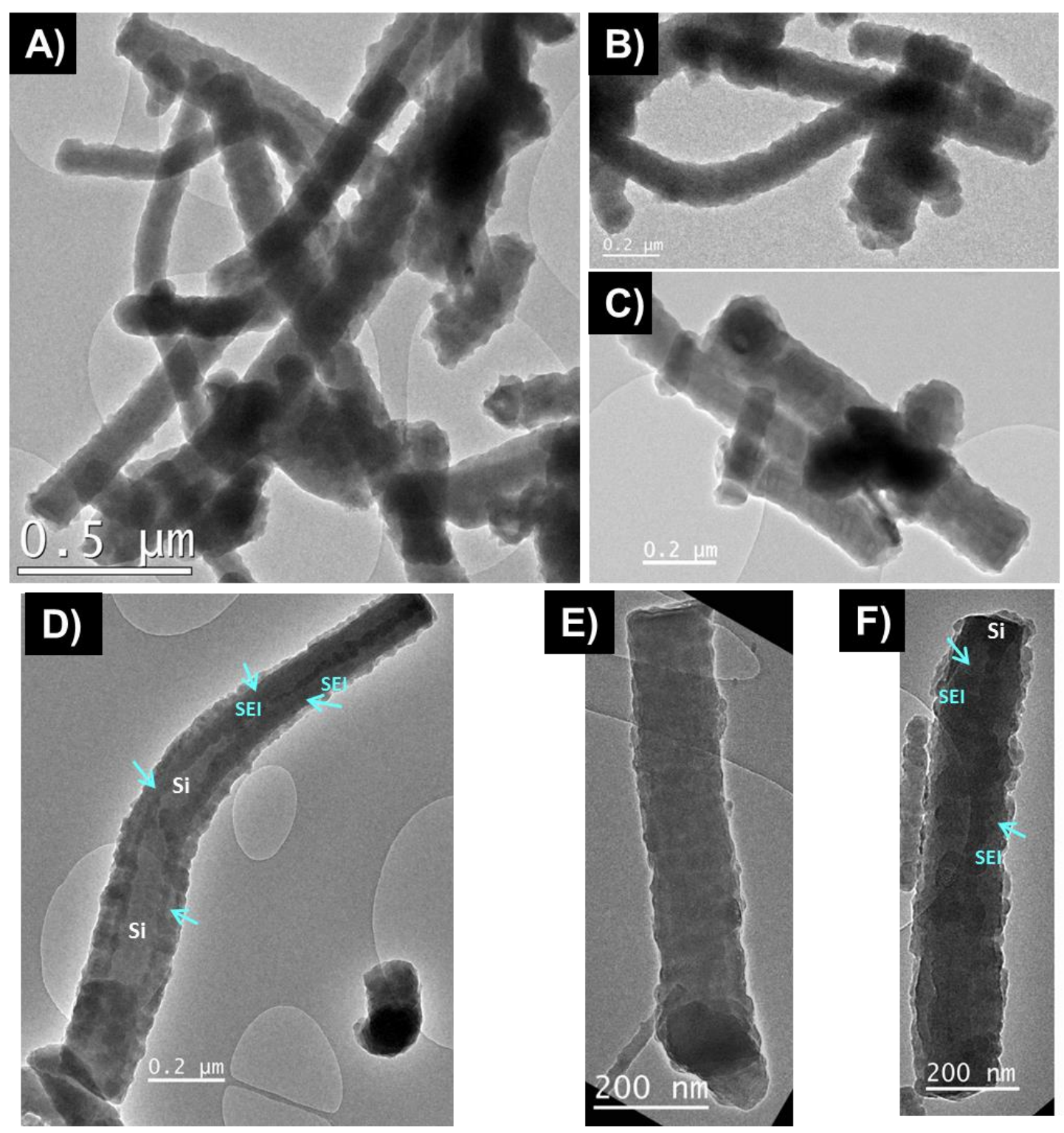

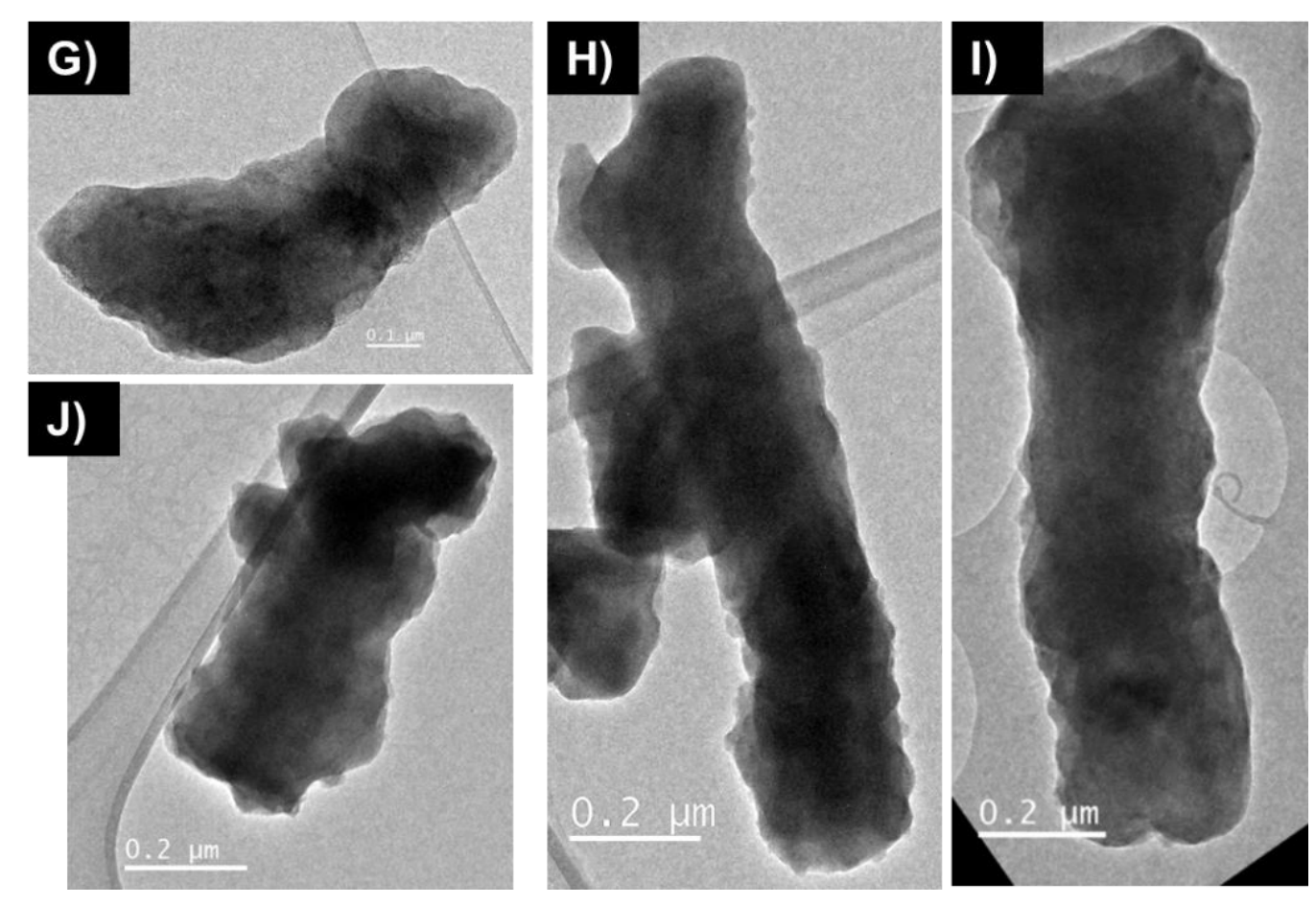

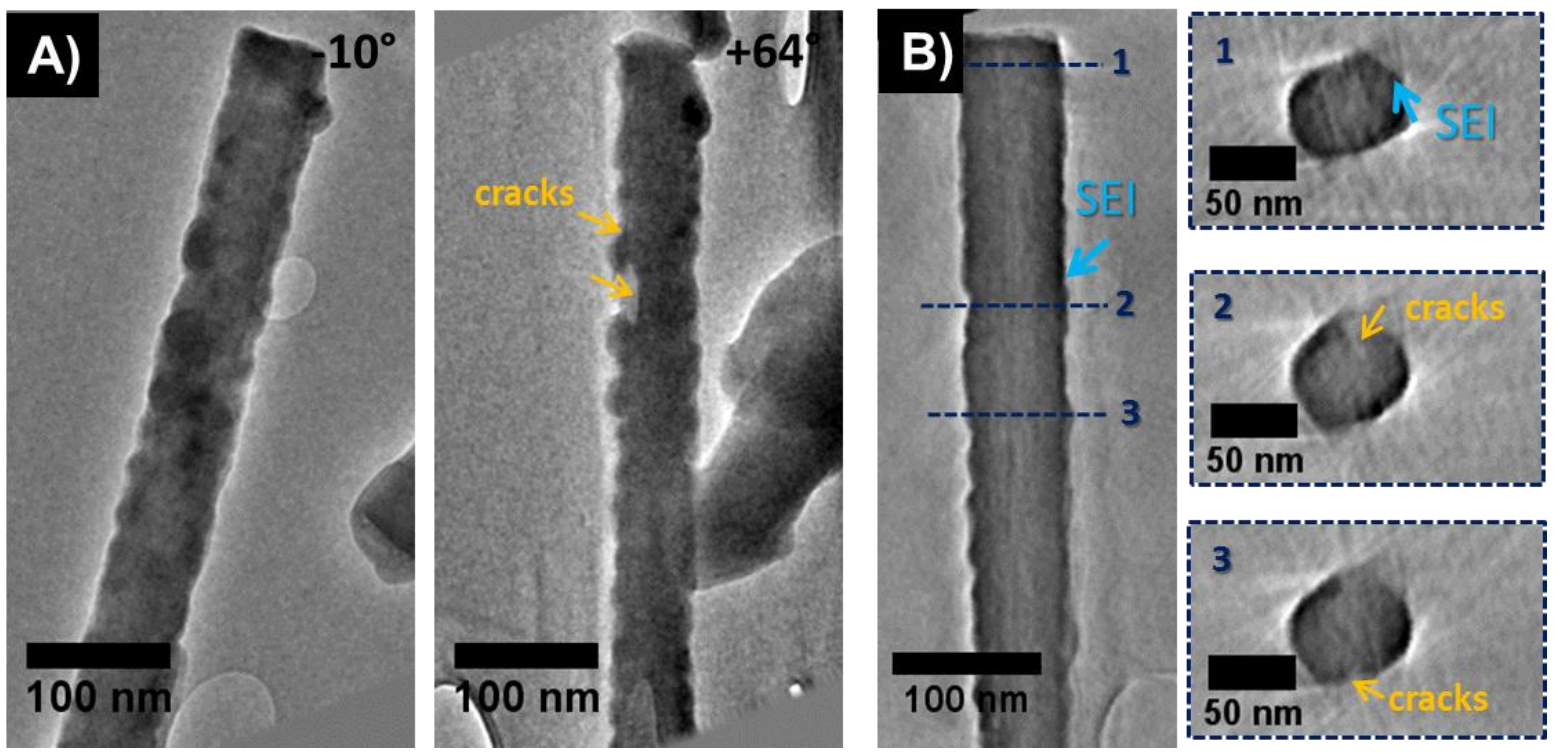

Electron Tomography analysis of SiNW cycled using a delithiation cut-off voltage of $0.6 \mathrm{~V}$ that undergo one cycle. A) Projections taken at two different tilt angles from the tilt series used to calculate the reconstructed volume. B) Transversal and longitudinal slices taken at different orientations of the reconstructed volume illustrating the homogenous distribution of the SEI all around the NWs with the presence of some micro-cracks.

\section{S4: STEM-EDS line scan analyses on an nanostructured Si NPs@VACNTs assembly obtained for 5 minutes exposure to $\mathrm{SiH} 4$ gas}
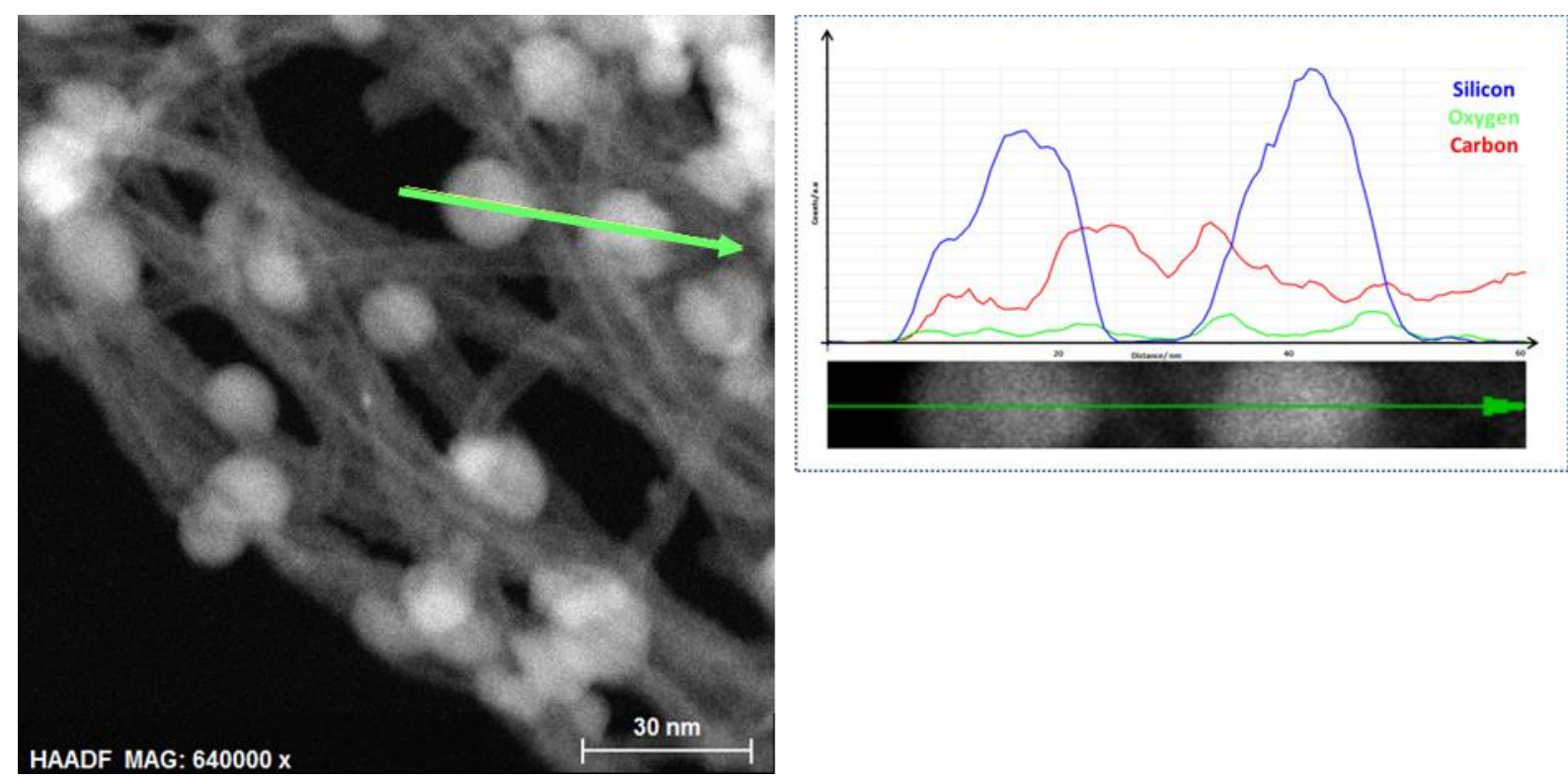

Figure S4: left) STEM-HAADF image of Si NPs@VACNT assembly; right top) HAADFSTEM image of Si NPs@VACNTs assembly where a line scan along the direction indicated by the green arrow was performed; right bottom) corresponding line-scan spectrum showing the variation of the $\mathrm{Si}$ (blue) $\mathrm{C}$ (red) and $\mathrm{O}$ (green) content along the scanning direction. 

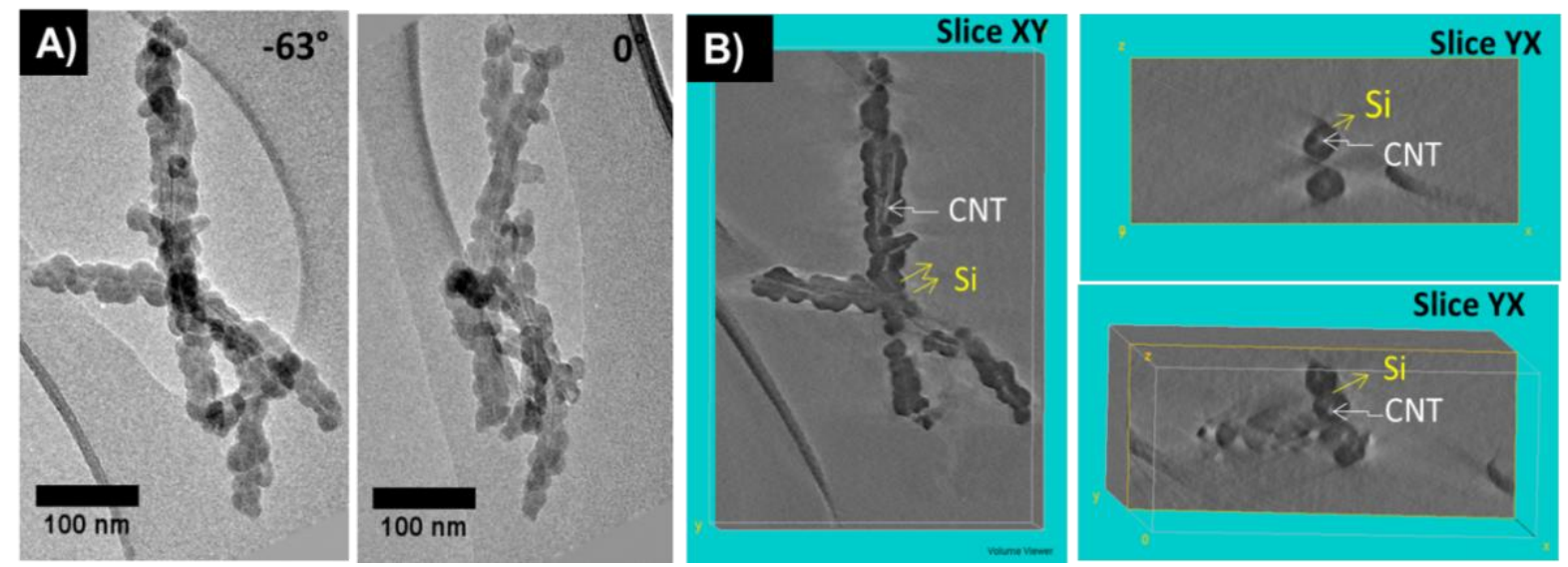

Figure S5: 3D reconstruction of a typical SiNPs@VACNTs sample that had undergone 10 minutes of exposure of the CNTs to the $\mathrm{SiH}_{4}$ precursor: a) 2D-TEM projections extracted from the tilt series used to calculate the object volume; b) typical ortho-slices extracted from the reconstructed volume.

S6: 3DTEM study of a typical SiNPs@VACNTs that undergone 15minutes of exposure of the CNTs to the $\mathrm{SiH} 4$ precursor
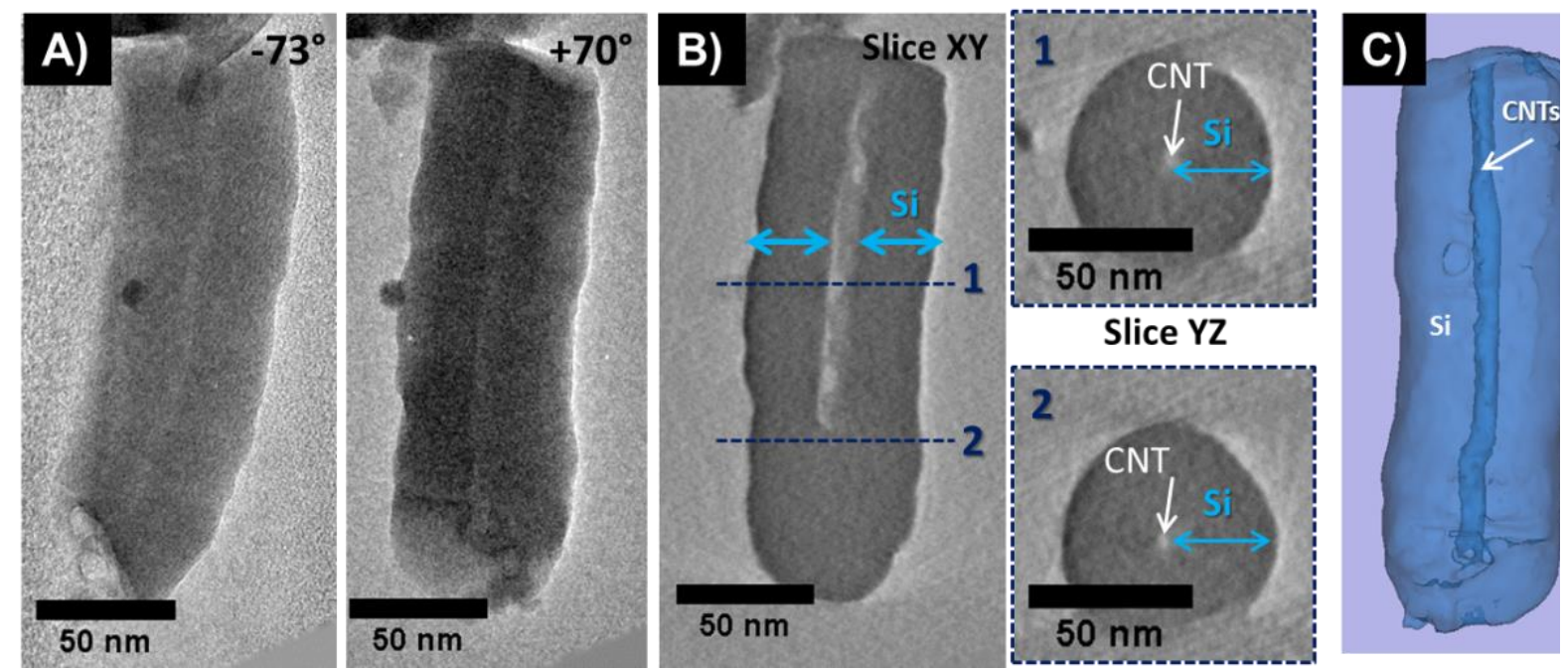

Figure S6: 3D Reconstruction of a typical Si NPs@VACNTs that undergone 15minutes of exposure of the CNTs to the SiH4 precursor: a) 2D-TEM projections extracted from the tilt series used to calculate the object volume; b) typical orthoslices extracted from the reconstructed volume; c) 3D model illustrating the nanostructure architecture obtained by data segmentation procedure.

\section{S7 : SEM micrographs on SiNPs@VACNTs carpets}




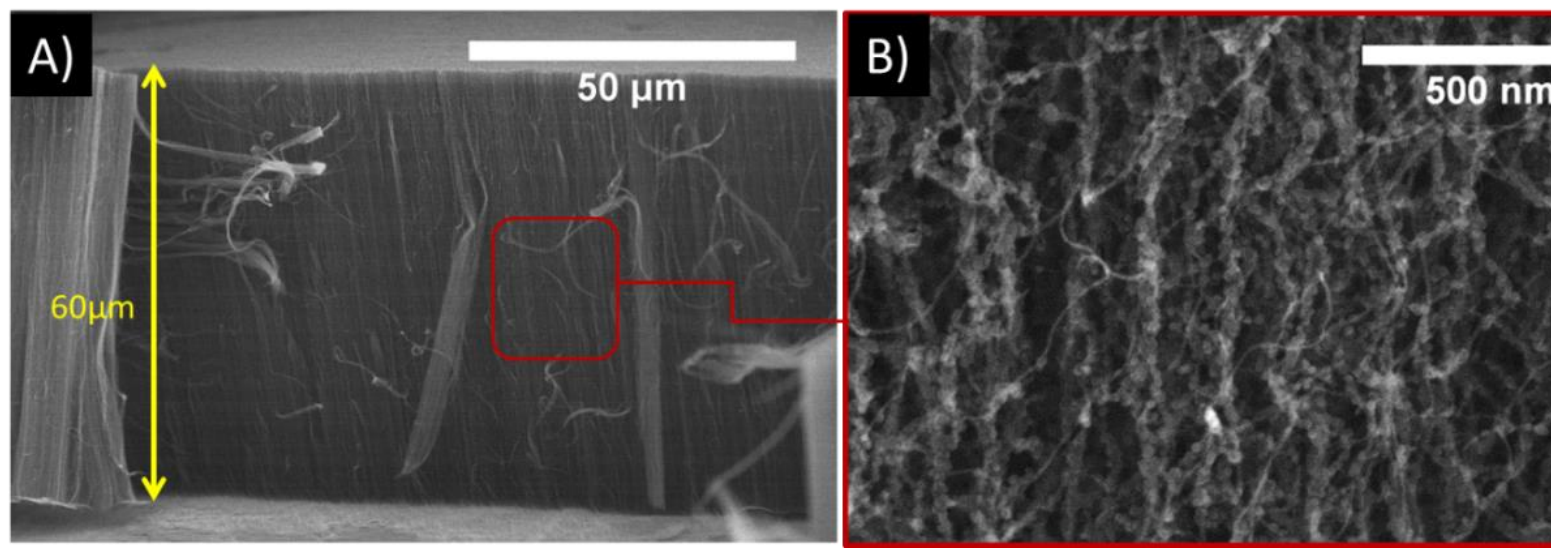

S8: Cycling at 1C of SiNPs @ VACNTs electrode vs Li in EC/DMC + LiPF6 1M + FEC 5 wt $\%$ between $20 \mathrm{mV}-2 \mathrm{~V}$.

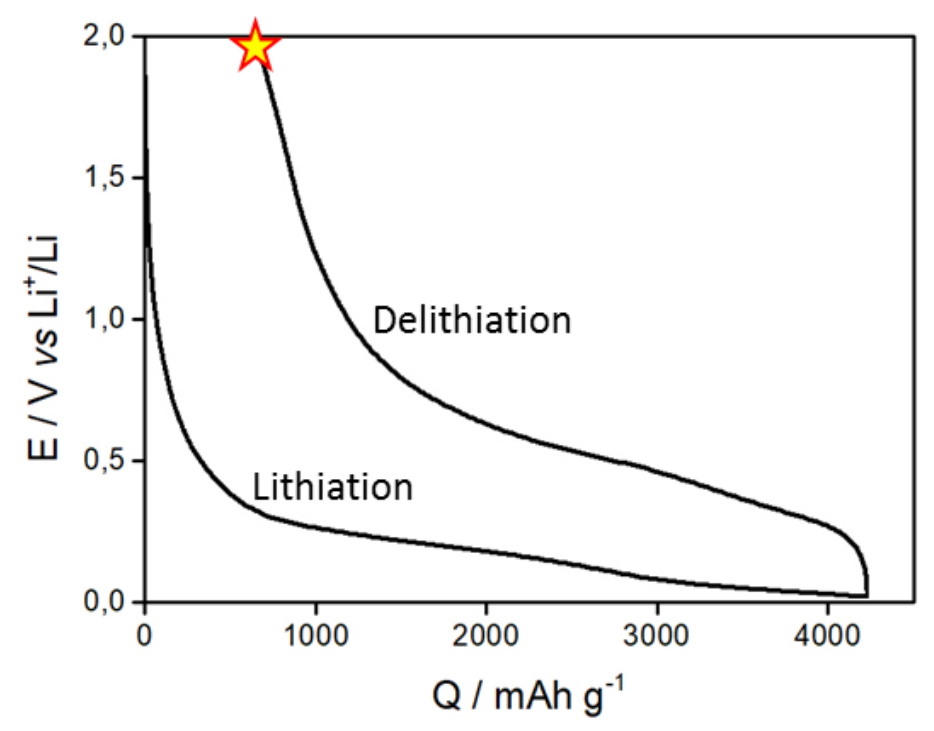




\section{S9: Electrochemical performances of SiNWs and SiNPs@CNTs anodes}

The electrochemical performances of SiNWs and SiNPs@VACNTs nanostructured electrodes were evaluated in half-cells. Galvanostatic lithiation/ delithiation curves between 0.02 and $2 \mathrm{~V}$ are shown in Figure S9A) and B). The mass of Si was determined before and after Si deposition was found to be $0.25 \mathrm{mg} \mathrm{cm}^{-2}$ and $0.3 \mathrm{mg} \mathrm{cm}^{-2}$ for SiNWs and SiNPs@VACNTs, respectively. For SiNWs (Figure S8A), the first lithiation and delithiation capacities were 4160 and $3236 \mathrm{mAh} \mathrm{g}^{-1}$, respectively, with a Coulombic efficiency of $78 \%$ at $\mathrm{C} / 20$ rate. From the second cycle onwards, a 1C rate was applied. The capacity rapidly drops to $2200 \mathrm{mAh} \mathrm{g}^{-1}$ due to the application of a higher current $(160 \mu \mathrm{A})$. Then, it slightly increases to reach about 2600 $\mathrm{mAh} \mathrm{g}^{-1}$ after 43 cycles, which can be attributed to the activation of more Si atoms reacting with $\mathrm{Li}^{+}$-ions. At the end of the $80^{\text {th }}$ cycle, a capacity close to $2270 \mathrm{mAh} \mathrm{g}^{-1}$ is obtained with a Coulombic efficiency of $99 \%$, which is more than six times that of commercial graphite anodes.

For SiNPs@VACNTs, the first Li insertion capacity was $4220 \mathrm{mAh} \mathrm{g}^{-1}$, while the first Li extraction capacity was $3620 \mathrm{mAh} \mathrm{g}^{-1}$, which yielded a first cycle coulombic efficiency of $86 \%$. Interestingly, SiNPs@VACNTs offered a cycling stability of $932 \mathrm{mAh} \mathrm{g}^{-1}$ over 80 cycles at a higher current of $1 \mathrm{~mA}$ compared to the $160 \mu \mathrm{A}$ of the SiNWs. The SiNPs @ VACNTs electrode delivered a specific capacity that is more than two times higher than the theoretical capacity of graphite. It has been reported that the capacity retention of cells operating at high C-rate is affected by the diffusion kinetics of charged particles that is influenced by the electrode thickness. For this reason, the electrode thickness is limited to $50 \mu \mathrm{m}$ in current high-power batteries.(W. Li, G. Zheng, Y. Yang, Z.W. Seh, N. Liu, Y. Cui, High-performance hollow sulfur nanostructured battery cathode through a scalable, room temperature, one-step, bottom-up approach., Proc. Natl. Acad. Sci. USA. 110 (2013) 7148-7153). The thickness of the SiNPs@VACNTs electrode used here is about $60 \mu \mathrm{m}$, and it is expected to show a similar stability even if its thickness significantly increases (i.e. of VACNTs were significantly longer), because the ions and electrons diffusion is ensured throughout the 1-D pathway. It is worth mentioning that conductive additives and even binders are avoided in this electrode fabrication method compared to the traditional one alleviating the weight of the battery. 

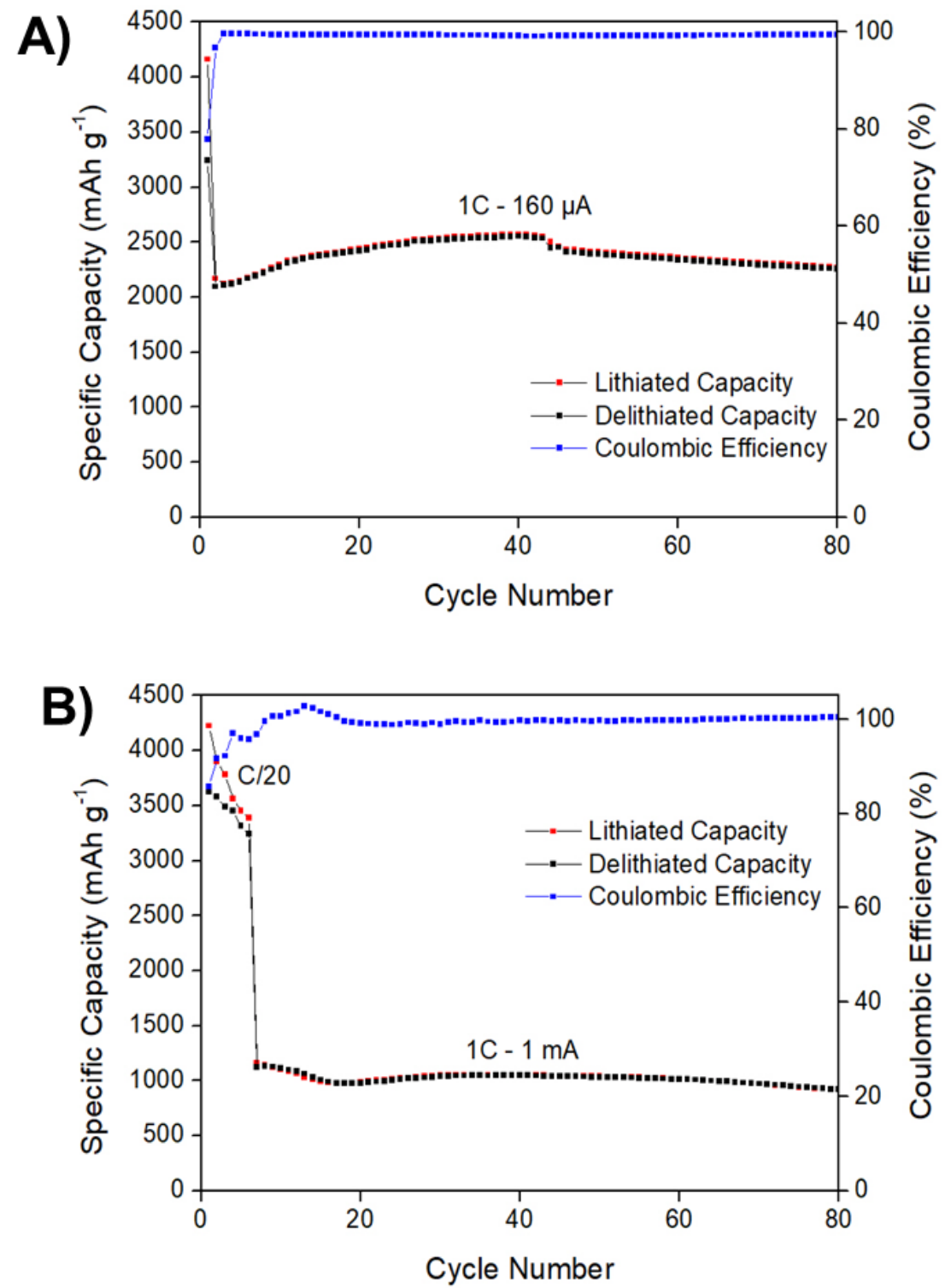

Figure S9. Lithiation/ delithiation capacities and Coulombic efficiency of SiNWs (A) and SiNPs@VACNTs (B) electrodes during 80 cycles. 